\title{
Extracting neural signals from semi-immobilized animals with deformable non-negative matrix factorization
}

\author{
Amin Nejatbakhsh ${ }^{1,2,3,4, *, \text {, }}$, Erdem Varol ${ }^{1,2,3,4,{ }^{*}}$, Eviatar Yemini ${ }^{5}$, Vivek Venkatachalam ${ }^{6}$, Albert Lin ${ }^{7}$, Aravinthan D.T. \\ Samuel $^{7}$, and Liam Paninski ${ }^{1,2,3,4}$ \\ ${ }^{1}$ Department of Neuroscience, Columbia University, New York, NY 10025 \\ ${ }^{2}$ Department of Statistics, Columbia University, New York, NY 10025 \\ ${ }^{3}$ Grossman Center for the Statistics of Mind, Columbia University, New York, NY 10025 \\ ${ }^{4}$ Zuckerman Institute, Center for Theoretical Neuroscience, Columbia University, New York, NY 10025 \\ ${ }^{5}$ Department of Biological Sciences, Columbia University, New York, NY 10025 \\ ${ }^{6}$ Department of Physics, Northeastern University, Boston, MA 02115 \\ Department of Physics, Harvard University, Cambridge, MA 02138
}

\begin{abstract}
Extracting calcium traces from populations of neurons is a 37 critical step in the study of the large-scale neural dynam- 38 ics that govern behavior. Accurate activity extraction re- ${ }_{39}$ quires the correction of motion and movement-induced deformations as well as demixing of signals that may overlap spatially due to limitations in optical resolution. Traditionally, non-negative matrix factorization (NMF) methods have been successful in demixing and denoising cellular calcium activity in relatively motionless or pre-registered videos. How- ${ }^{44}$ ever, standard NMF methods fail in animals undergoing sig- 45 nificant non-rigid motion; similarly, standard image registra- 46 tion methods based on template matching can fail when large 47 changes in activity lead to mismatches with the image tem- ${ }_{48}$ plate. To address these issues simultaneously, we introduce a deformable non-negative matrix factorization (dNMF) framework that jointly optimizes registration with signal demixing. On simulated data and real semi-immobilized $C$. elegans microscopy videos, dNMF outperforms traditional demixing meth- ${ }^{52}$ ods that account for motion and demixing separately. Finally, ${ }^{53}$ following the extraction of neural traces from multiple imaging ${ }^{54}$ experiments, we develop a quantile regression time-series nor- 55 malization technique to account for varying neural signal inten- 56 sity baselines across different animals or different imaging se- 57 tups. Open source code implementing this pipeline is available 58 at https://github.com/amin-nejat/dNMF.
\end{abstract}

\section{Introduction}

Recent advances in imaging techniques have enabled the capture of functional neural ensembles in vivo within ${ }^{64}$ a wide variety of animal models [Flusberg et al., 2008, ${ }^{65}$ Ahrens et al., 2013, Prevedel et al., 2014, Mann et al., 2017]. ${ }^{6}$ Demixing the recorded video signals into estimates of indi- ${ }^{67}$ vidual neural activity remains a critical bottleneck in the anal- ${ }^{68}$ ysis of these large and complex datasets. Previous approaches ${ }^{69}$ for extracting individual neural activity traces have involved either region of interest (ROI) methods [Kerr et al., 2005, Niell and Smith, 2005,

Dombeck et al., 2007,

* Equally contributing authors

Correspondence: mn2822@columbia.edu
Göbel et al., 2007, Tian et al., 2009, Kerlin et al., 2010, Hofer et al., 2011,

Venkatachalam et al., 2016b, Nguyen et al., 2016, Barbera et al., 2016] or matrix factorization methods based on principal components analysis (PCA) or independent components analysis (ICA) [Stetter et al., 2001, Siegel et al., 2007, Reidl et al., 2007, Mukamel et al., 2009] or sparse coding [Pachitariu et al., 2013, Pachitariu et al., 2017].

Non-negative matrix factorization

(NMF) [Paatero and Tapper, 1994, Lee and Seung, 1999, Lee and Seung, 2001] based models have been introduced to demix signals from recordings of calcium activity [Andilla and Hamprecht, 2013, Haeffele et al., 2014, Andilla and Hamprecht, 2014, Maruyama et al., 2014, Pnevmatikakis et al., 2016, Pachitariu et al., 2017, Zhou et al., 2018]. A prerequisite for the success of these methods, to permit blind-source separation, is that the imaged ROI remains motionless even when the animal is awake, satisfying the assumption that the spatial footprints of signal sources remain stationary. To facilitate NMF assumptions and remove excess motion variability, a common pre-processing step before NMF is the registration of the imaging volumes to a common template space.

There is a wealth of literature in the medical imaging community regarding the registration of volumetric images to template volumes to account for morphological variability [Klein et al., 2009]. These methods have proven to be very effective in registering images that have similar intensity profiles but they tend to introduce artifacts when the template image and the moving image have different appearances, low signal to noise ratio, or abnormalities [Zeng et al., 2016]. Furthermore, the computational complexity of these methods is a bottleneck since there are potentially tens of thousands of frames in volumetric calcium videos that need to be registered. A number of pipelines [Dubbs et al., 2016, Pnevmatikakis and Giovannucci, 2017, Pachitariu et al., 2017] implement existing sub-pixel registration techniques [Guizar-Sicairos et al., 2008] to enable the rigid and non-rigid registration of calcium videos in a com- 
putationally efficient manner. Assuming that the motion does 133 not involve large shifts in the field of view (FOV), these tech-134 niques aim to register individual video frames to a template ${ }_{135}$ frame through fast patchwise rigid transformations. However, ${ }_{136}$ they too are not built to handle severe deformations and large ${ }_{137}$ intensity variations.

Recent whole-brain imaging techniques of the 139 model organism C. elegans [Schrödel et al., 2013,140 Prevedel et al., 2014, Kato et al., 2015, Nguyen et al., 2016,141 Venkatachalam et al., 2016b] have opened up an exciting 142 new avenue of research, enabling simultaneous recording 143 of neural dynamics and freely-moving behaviors in the 144 same animal. Even during restrained imaging, worms ${ }_{145}$ can exhibit highly-nonlinear motion [Girard et al., 2007, ${ }_{146}$ Larsch et al., 2013, Voleti et al., 2019], violating the as- ${ }_{147}$ sumptions that enable NMF-based signal separation and ${ }_{148}$ overstretching the capabilities of fast piecewise rigid registra- ${ }_{149}$ tion techniques. Therefore, common approaches have been ${ }_{150}$ to apply motion tracking and simple pixel-averaging around ${ }_{151}$ cellular tracking ROIs in two discrete steps, often followed ${ }_{152}$ by time-consuming supervision and manual correction of ${ }_{153}$ the results [Kato et al., 2015, Venkatachalam et al., 2016a, ${ }_{154}$ Nguyen et al., 2016]. One way to perform motion tracking ${ }_{155}$ is to use a second imaging channel to record a temporally- ${ }_{156}$ invariant fluorescent marker (such as RFP) which is ${ }_{157}$ insensitive to calcium activity. By using such cellular motion ${ }_{158}$ tracking markers, calcium activity can then be extracted by ${ }_{159}$ averaging the pixel values in the ROI that overlap with the ${ }_{160}$ marker. However, this approach is flawed for at least two ${ }_{161}$ reasons: 1) ROI averaging in densely-packed cell regions ${ }_{162}$ is prone to mixing signal between different neurons, due ${ }_{163}$ to limitations in optical resolution, and 2) introducing $\mathrm{a}_{164}$ second imaging channel effectively requires experimenters ${ }_{165}$ to reduce the frame rate and/or spatial resolution by at least ${ }_{166}$ half in order to acquire this channel or add an additional ${ }_{167}$ optical path and camera. On the other hand, if tracking is ${ }_{168}$ performed only on the calcium imaging channel, due to the ${ }_{169}$ low signal-to-noise regime and calcium signal fluctuations, ${ }_{170}$ tracking approaches may miss cellular markers at time points ${ }_{171}$ when the cells become dim, creating downstream errors in 172 tracking and demixing.

In general, tracking cells in moving animals (and even re-174 strained animals with restricted mobility), has proven to be ${ }_{175}$ a challenging machine vision problem [Hirose et al., 2017].176 Cell nuclei have similar shapes, thus providing only a lim- ${ }_{177}$ ited set of unique features to facilitate their tracking. Spatial ${ }_{178}$ noise represents a further, inherent limitation, due to the mi- ${ }_{179}$ croscopic size of the objects under investigation. Most avail- ${ }_{180}$ able microscopy approaches scan the animal in both space $_{181}$ and time to achieve volumetric video recordings. Therefore, ${ }_{182}$ there are fundamental limits in reaching the high spatiotem-183 poral resolution necessary to resolve unique cell identities ${ }_{184}$ and extract their calcium signals through tracking techniques. 185

Even if high accuracy cell tracking can be achieved, an-186 other issue with extracting calcium signal around tracked ${ }_{187}$ ROIs is that many existing volumetric optical imag-188 ing setups have a relatively poor resolution in the ${ }_{189}$ depth axis, characterized by an elongated point-spreadfunction [Yang and Yuste, 2017]. This phenomenon causes the calcium signals of nearby cells to be mixed, which in turn causes the pixel-wise signal read-out to be an inaccurate portrayal of actual neural activity.

Orthogonally, there have been NMF techniques that are invariant to signal shift, such as convolutive NMF [O'grady and Pearlmutter, 2006, Smaragdis, 2006]. However, these techniques model discrete translation based shifts and are not suitable for modeling the complex deformable motion exhibited across biological volumetric recordings.

In the case of $C$. elegans imaging, worms can exhibit nonlinear motion (even when immobilized using popular paralytics [Larsch et al., 2013, Venkatachalam et al., 2016a, Voleti et al., 2019]) and variability in their neural firing patterns over time, making the application of previous techniques such as Normcorre [Pnevmatikakis and Giovannucci, 2017] or convolutive NMF ineffective. To surmount these issues, we introduce deformable non-negative matrix factorization (dNMF) to jointly model the motion, spatial shapes, and temporal traces of the observed neurons in a tri-factorization framework. Instead of the two-step approach of sequentially tracking then demixing calcium signals, we update motion parameters together with updates in the spatial and temporal matrices. To ensure that our model is not overfitting and picking up spurious motion and signal, we use regularized models for cell shapes, temporal fluctuations, and deformations. The model parameters capture the worm's motion corresponding to a fixed, spatial representation of the video, enabling the deformation terms to match the worm's posture at each time frame. Our framework is general and is suitable for decomposing videos into a set of motion parameters, fixed spatial representations for image components, and temporally varying signals with underlying linear and/or nonlinear motion. This approach can be considered a generalization of the model developed in [Peng et al., 2012] (applied to calcium imaging data by [Poole et al., 2015]), which restricts attention to affine transformations.

We validate our method on an intensity-varying particle-tracking simulation and compare it to stateof-the-art calcium-imaging motion-correction techniques [Pnevmatikakis and Giovannucci, 2017] followed by NMF [Pnevmatikakis et al., 2016]. We then demonstrate the ability of our framework to extract calcium traces from all neurons in the head and tail of semi-immobilized C. elegans exhibiting nonlinear motion. We use a dataset of 42 animals, 21 worm heads and 21 worm tails, recorded for 4 minutes each while presenting three stimuli, a repulsive concentration of salt and two attractive odors. We find that the proposed approach outperforms both ROI averaging and standard $\mathrm{NMF}$, delivering more accurate tracking and demixing than either of these methods in this dataset.

Finally, after accurate extraction of neural activity signals from each animal, a post-processing normalization step is still required in order to compare neurons of the same type, 
across a population of animals. This is because factors such 230 as variable illumination, anisotropy associated with animal ${ }_{231}$ orientation, and a lack of stereotypy in fluorescence expres-232 sion across animals introduce substantial variability into the ${ }_{233}$ baseline and amplitude of the extracted neural activity sig-234 nals. Standard post-processing approaches based on esti-235 mating $\Delta F / F_{0}$, do not resolve this variability, which if un-236 corrected will confound any group-type neural comparisons ${ }_{237}$ across animals. Even worse, outlier signals that arise due to mistracking and demixing can considerably warp the mean ${ }^{238}$ signal measured across a population of animals, especially when the neuron type of interest is dim and is present next to neuron types with brighter signal.

To reduce this excess variability across animals, we introduce a time-series normalization approach, termed quantile regression. This approach optimizes for a linear transformation of time-series intensities in a group of samples (e.g., all traces extracted from a given cell type over all animals), transforming the time-series samples to have matched histograms. We compare this approach with z-scoring and advocate its ${ }_{239}$ adoption for population-based time-series analysis due to sev- ${ }_{240}$ eral desirable properties. In particular, our approach retains ${ }_{241}$ the approximate baseline and magnitude across a population ${ }_{242}$ of neurons of the same type, while maintaining robustness ${ }_{243}$ against outlier signals. Lastly, we introduce an option for en- ${ }_{244}$ suring the non-negativity of the normalized signals, when ap- ${ }_{245}$ propriate for the biological measurements being performed.

\section{Methods}

The joint motion correction and signal extraction framework proposed here involves several steps illustrated in Figure $1 .{ }^{249}$ First, the volumes undergo several pre-processing steps that ${ }^{250}$ involve coarse tracking, background subtraction and smoothing, details of which are discussed in the "Pre-processing steps" subsection below. The pre-processed volumes are then subjected to simultaneous deformation compensation and signal demixing using a matrix tri-factorization model.

First, we introduce notation. Let $\boldsymbol{Y}_{t} \in \mathbb{R}^{d}$ denote the $d$ pixel vectorized volumetric image at time $t=1, \ldots, T$. We seek to decompose the observations, $\boldsymbol{Y}_{t}$, into a factorization involving a time-varying deformation term, $\boldsymbol{f}_{\beta_{t}}$ that acts on a time-invariant canonical representation of $k$ object shapes encoded by $\boldsymbol{A}$. The time-varying spatial signatures, $\boldsymbol{f}_{\beta_{t}}(\boldsymbol{A}) \in \mathbb{R}^{d \times k}$, are then multiplied by signal carrying coefficients $\boldsymbol{C}_{t} \in \mathbb{R}^{k}$. We also encourage model parameters to be "well-behaved" using regularization functions, $\mathcal{R}$ (details of which will be outlined later). The resulting objective function is:

$$
\begin{aligned}
& \min _{\boldsymbol{A}, \boldsymbol{C}, \boldsymbol{\beta}} \sum_{t=1}^{T}\left\|\boldsymbol{Y}_{t}-\boldsymbol{f}_{\boldsymbol{\beta}_{t}}(\boldsymbol{A}) \boldsymbol{C}_{t}\right\|_{2}^{2}+\mathcal{R}(\boldsymbol{A}, \boldsymbol{C}, \boldsymbol{\beta}) \\
& \text { s.t. } \boldsymbol{A}, \boldsymbol{C}_{1: T} \geq 0
\end{aligned}
$$

This formulation differs from standard NMF techniques [Lee and Seung, 2001] in that the spatial footprint term consists of a time invariant term, $\boldsymbol{A}$ and a time vary-251 ing term, $\boldsymbol{f}_{\boldsymbol{\beta}_{t}}$, which is a differentiable transformation 252 parametrized by $\boldsymbol{\beta}_{t}$, that deforms the canonical representation into the $t$-th time frame. $\boldsymbol{\beta}_{t}$ encapsulates the motion parameters and is usually low dimensional to avoid over-parameterization and overfitting. The regularization $\mathcal{R}(\cdot)$ further constrains the possible choice of spatial footprints, signal coefficients, and spatial deformations. Figure 1 illustrates the model. Next, we detail two possible parameterizations of the spatial terms, $\boldsymbol{A}$ and $\boldsymbol{f}$.

\subsection{Spatial component: non-parametric model}

Similar to the standard NMF models, we can parameterize $\boldsymbol{A}$ using a $d$-by- $k$ matrix, where $d$ is the number of pixels of one time frame of the video and $k$ is the number of objects that are present. We use a Gaussian interpolant, $\boldsymbol{T}_{t}$, to transform these spatial footprints to arbitrary locations such that $\boldsymbol{f}_{\boldsymbol{\beta}_{t}}(\boldsymbol{A})=\boldsymbol{T}_{t} \boldsymbol{A}$, where $\boldsymbol{T}_{t}: \mathbb{R}^{d \times d}$ and

$$
\boldsymbol{T}_{t}[i, j]=\exp \left(\frac{\left\|\boldsymbol{\beta}_{t} \Psi\left(\boldsymbol{x}_{j}\right)-\boldsymbol{x}_{i}\right\|_{2}^{2}}{2 \sigma^{2}}\right) .
$$

Here, $\boldsymbol{x}_{\boldsymbol{i}}, \boldsymbol{x}_{\boldsymbol{j}} \in \mathbb{R}^{3}$ denote the coordinates of two arbitrary pixels in the volume. $\Psi: \mathbb{R}^{3} \rightarrow \mathbb{R}^{p}$ denotes a basis mapping of coordinates to enable non-linear deformations and $\boldsymbol{\beta}_{t}$ is a 3 -by- $p$ matrix that parametrize the deformations. For example, in the case of a quadratic polynomial basis, $\boldsymbol{\beta}_{t}$ would be a 3-by-10 matrix, and $\Psi: \mathbb{R}^{3} \rightarrow \mathbb{R}^{10}$ would be the quadratic basis function $\Psi\left([x, y, z]^{T}\right)=\left[1, x, y, z, x^{2}, y^{2}, z^{2}, x y, y z, x z\right]^{T}$. The choice of $\sigma$ controls the amount of the spread of the mass of a pixel into nearby pixels. Further details on optimization in this model can be found in the appendix 1 .

\subsection{Spatial component parametrization: Gaussian functions}

When we have strong prior information about the component shapes we can incorporate that into the model using an appropriate parameterization for the spatial footprints. Neural activity is most commonly imaged using cytosolic or nuclearlocalized calcium indicators; nuclear-localized indicators can be reasonably modelled using ellipsoidally-symmetric shape models. Specifically, we observed that the spatial component of the neurons in the videos analyzed here, of $C$. elegans imaged using nuclear-localized calcium indicators, can be well approximated using three-dimensional Gaussian functions. By taking advantage of this observation we can reduce the number of parameters in $\boldsymbol{A}$ from one parameter per pixel per component, to $k 3 \mathrm{D}$ centers ( 3 parameters per each neuron) and $k$ covariance matrices (6 parameters per each neuron using the Cholesky parameterization). Formally, we model the footprint of component $k$ using a 3-dimensional Gaussian function with location parameters $\boldsymbol{\mu}_{k} \in \mathbb{R}^{3}$ and shape parameters $\boldsymbol{\Sigma}_{k} \in \mathbb{R}^{3 \times 3}$. Under this new spatial model for $\boldsymbol{A}=\left\{\boldsymbol{\mu}_{1: K}, \boldsymbol{\Sigma}_{1: K}\right\}$, we modify the $\boldsymbol{f}_{\boldsymbol{\beta}_{t}}$ function to match this parameterization to have $\boldsymbol{f}_{\boldsymbol{\beta}_{t}}(\boldsymbol{A}) \in \mathbb{R}^{d \times k}$ :

$$
\boldsymbol{f}_{\boldsymbol{\beta}_{t}}(\boldsymbol{A})[i, k] \approx \exp \left(\left[\boldsymbol{p}_{i}-\boldsymbol{\beta}_{t} \Psi\left(\boldsymbol{\mu}_{k}\right)\right]^{T} \boldsymbol{\Sigma}_{k}^{-1}\left[\boldsymbol{p}_{i}-\boldsymbol{\beta}_{t} \Psi\left(\boldsymbol{\mu}_{k}\right)\right]\right),
$$

where $\boldsymbol{p}_{i}$ is the 3D coordinate of the $i$-th pixel in the image. (Note that non-negativity of the spatial components is 

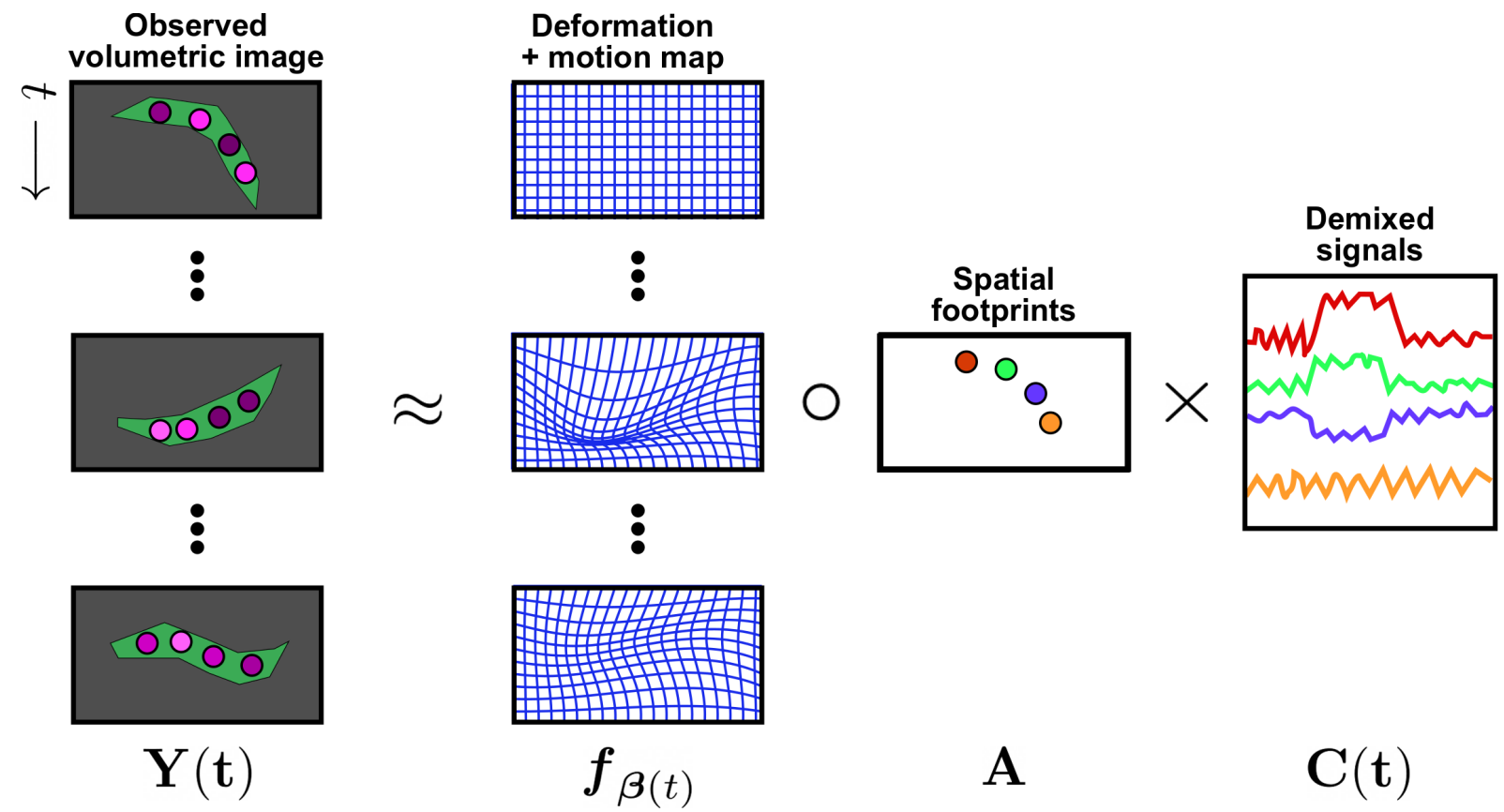

Fig. 1. Schematic of the deformable non-negative matrix factorization model. The volumetric time series data $Y(t)$ is factorized into time-varying deformation + motion maps $\boldsymbol{f}_{\boldsymbol{\beta}(t)}$ which transform the factorized signal (with spatial footprints $\boldsymbol{A}$ multiplied by time-varying intensity coefficients, $\boldsymbol{C}(t)$ ) onto the observed data volumes. $\boldsymbol{f}_{\boldsymbol{\beta}_{t}}$ induces a deformable transformation of the pixel correspondences between time $t$ and the canonical representation $\boldsymbol{A}$. In order to constrain this transformation to yield physically realistic deformations that respect volumet-275 ric changes, we regularize the cost function using the deter-276 minant of the Jacobian of the transformation term to encour-277 age the Jacobian to be close to 1 and prevent the deformation 278 from contracting or expanding unrealistically. The Jacobian 279

can be represented as:

$$
\mathcal{J}_{\boldsymbol{\beta}}\left(x_{1}, x_{2}, x_{3}\right) \quad \text { with } \quad \mathcal{J}_{i j}=\frac{\partial\left(\boldsymbol{f}_{\boldsymbol{\beta}}\right)_{i}}{\partial x_{j}} .
$$

Using the Jacobian, the regularizer is:

$$
\mathcal{R}_{\mathcal{J}}(\boldsymbol{\beta})=\lambda_{\mathcal{J}} \sum_{t=1}^{T} \sum_{i=1}^{j}\left(\operatorname{det} \mathcal{J}_{\boldsymbol{\beta}_{t}}\left(x_{i}, y_{i}, z_{i}\right)-1\right)^{2}
$$

$$
\begin{aligned}
\mathcal{R}_{T}(\boldsymbol{C}, \boldsymbol{\beta})= & \lambda_{\beta} \sum_{t=0}^{T-1}\left\|\psi\left(\boldsymbol{\mu}_{1: K}\right) \boldsymbol{\beta}_{t-1}-\psi\left(\boldsymbol{\mu}_{1: K}\right) \boldsymbol{\beta}_{t}\right\|_{F}^{2} \\
& +\lambda_{C} \sum_{t=0}^{T-1}\left\|\boldsymbol{C}_{t-1}-\boldsymbol{C}_{t}\right\|_{F}^{2}
\end{aligned}
$$

\subsection{Regularization: Jacobian constraints for plausible deformations}

$$
\text { in [ }
$$

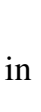

where the Jacobian is evaluated on a grid where we want to ensure its proximity to one.

\subsection{Optimization}

All the variations of the dNMF cost function are optimized in the following way. To update $\boldsymbol{\beta}$ and $\boldsymbol{A}$ we use the autograd tool and PyTorch library to automatically compute gradients of the cost function and Adam optimizer to back-propagate the gradients. A forward pass of computation is evaluating the cost function with $\boldsymbol{\beta}_{1: T}$ and $\boldsymbol{A}$ (in the fully parametric case, or $\boldsymbol{\beta}_{1: T}$ (in the Gaussian case) as parameters. Note that for a fixed $\boldsymbol{C}$, all compartments of the cost function are differentiable with respect to the parameters.

To update $\boldsymbol{C}$ we use multiplicative updates as described [Taslaman and Nilsson, 2012]:

$$
\boldsymbol{C}_{t} \leftarrow \boldsymbol{C}_{t} \odot \frac{\boldsymbol{f}_{\boldsymbol{\beta}_{t}}^{T} \boldsymbol{Y}_{t}+\lambda_{C}\left(\boldsymbol{C}_{t-1}+\boldsymbol{C}_{t+1}\right)}{\boldsymbol{f}_{\boldsymbol{\beta}_{t}}^{T} \boldsymbol{f}_{\boldsymbol{\beta}_{t}} \boldsymbol{C}_{t}+2 \lambda_{C} \boldsymbol{C}_{t}} .
$$

The key difference between these multiplicative updates from those found in [Lee and Seung, 2001] is that the parts of the derivatives of the temporal smoothness regularization terms $2 \lambda_{C} \boldsymbol{C}_{t}$ and $\lambda_{C}\left(\boldsymbol{C}_{t-1}+\boldsymbol{C}_{t+1}\right)$ appear in the denominator and numerator to promote smoothly varying signal. 


\subsection{Initialization}

One key advantage of the $C$. elegans datasets considered 315 here is that we can reliably identify the locations of all ${ }^{316}$ cells in the field of view, using methods developed in 317 [Yemini et al., 2019]. Using the location of cells in the ini-318 tial frame (for example) can tremendously aid the optimiza-319 tion of the objective 1 for two main reasons. First, it serves ${ }^{320}$ as a very good initializer for the $\mu_{k}$ parameters for cell spa-321 tial footprints mentioned in section 2. Second, we know ${ }^{322}$ a priori the correct number of cells to be demixed in the ${ }^{323}$ FOV. These two factors enable our framework to operate ${ }^{324}$ in a semi-blind manner towards the deconvolution of neu-325 ral signals of $C$. elegans, unlike fully blind deconvolution techniques such as e.g. PCA-ICA [Mukamel et al., 2009] or $_{326}$ CNMF [Pnevmatikakis et al., 2016].

\subsection{Using dNMF for image registration}

The transformation terms $\boldsymbol{f}_{\boldsymbol{\beta}_{\boldsymbol{t}}}$, learned using dNMF, can ${ }^{329}$ be used to obtain a pixel-level transformation of the video ${ }^{330}$ frames to a reference frame in order to yield a registered ${ }^{33}$ video; in the ideal case this registered video would remove all ${ }^{332}$ the motion from the video, leaving each neuron to flicker in ${ }^{333}$ place as its internal activity modulates its fluorescence level. ${ }^{334}$ In the current formulation, $\boldsymbol{f}_{\boldsymbol{\beta}_{\boldsymbol{t}}}$ represent push-forward map- ${ }^{335}$ pings of a reference frame to all the frames in the video. How- ${ }^{336}$ ever, to obtain a registration, we need to recover the inverse ${ }^{337}$ mappings from all of the video frames to the reference frame. ${ }^{338}$ We solve this inverse transform, $\boldsymbol{\beta}_{t}^{i}$, by optimizing the follow- ${ }_{340}$ ing objective:

$$
\min _{\boldsymbol{\beta}_{t}^{i}} \sum_{t=1}^{T}\left\|\boldsymbol{\mu}_{1: K}-\boldsymbol{\beta}_{t}^{i} \boldsymbol{\psi}\left(\boldsymbol{\beta}_{t}^{*} \boldsymbol{\psi}\left(\boldsymbol{\mu}_{1: K}\right)\right)\right\|_{F}^{2}+\mathcal{R}_{\mathcal{J}}\left(\boldsymbol{\beta}^{\boldsymbol{i}}\right)
$$
where $\boldsymbol{\mu}_{1: K} \in \mathbb{Z}_{+}^{K \times 3}$ indicates the set of neuron coordinates ${ }^{345}$ at the reference frame and $\boldsymbol{\beta}_{t}^{*} \boldsymbol{\psi}\left(\boldsymbol{\mu}_{1: K}\right)$ indicates the forward ${ }^{346}$ polynomial mapping of these neurons in the $t$-th video frame 347 after optimization, with $\boldsymbol{\beta}_{t}^{*}$ indicating the transformation op-348 timized through Eq. (1). Lastly, $\mathcal{R}_{\mathcal{J}}(\boldsymbol{\beta})$ indicates the same 349 Jacobian regularizer as in Eq. (6). In the simplest case that 350 $\beta_{t}^{*} \boldsymbol{\psi}$ is restricted to be affine, and the regularizer weight $\lambda_{J}{ }^{351}$ in $\mathcal{R}_{\mathcal{J}}\left(\boldsymbol{\beta}_{\boldsymbol{t}}^{\boldsymbol{i}}\right)$ is negligible, then $\boldsymbol{\beta}_{t}^{i} \boldsymbol{\psi}$ simply implements the ${ }_{352}$ shift and matrix inversion of $\beta_{t}^{*} \boldsymbol{\psi}$. More generally, the exact ${ }_{353}$ inverse mapping may not exist or may be unstable; in this 354 more general setting Eq. (8) will output a smooth approxima-355 tion to the inverse mapping.

Note that Eq. (8) solves a labeled point-set registration 357 problem (since it operates on the neuron centers $\boldsymbol{\mu}_{1: K}$ ), not ${ }_{358}$ an image registration problem per se. Next we use the recov- 359 ered inverse mapping $\boldsymbol{\beta}_{t}^{i}$ to perform image registration, using ${ }_{360}$ pixel-wise interpolation:

$$
\boldsymbol{p}_{t} \mapsto \operatorname{Interp} .\left[\boldsymbol{\beta}_{t}^{i} \boldsymbol{\psi}\left(\boldsymbol{p}_{t}\right)\right] .
$$

Here, $\boldsymbol{p}_{t} \in \mathbb{Z}_{+}^{d \times 3}$ denotes the mesh of pixel coordinates that ${ }_{364}$ span the entire volume of the image and Interp. refers to an in-365 terpolation function such as linear, nearest neighbor, or bicu- 366 bic, that can be used to convert non-integer values of pixel co-367 ordinates to map to discrete pixels. In practice, we set the ref- -368 erence frame to be the first frame in the video series and use ${ }_{369}$ linear interpolation. Note that this way of performing registration differs from traditional registration techniques such as Normcorre [Pnevmatikakis and Giovannucci, 2017] in a critical way: the deformation terms that are used to drive the registration are informed by the neural activity and are decoupled from the inferred activity in the joint objective function Eq. (1). Thus, in theory, large fluctuations in neural activity from frame to frame should not affect the deformation terms. In contrast, pure registration techniques on functional neural data may be driven to poor local optima if the neural activity in a particular frame differs strongly from the reference frame.

\subsection{Population neural analysis}

After we have extracted activity traces from each neuron in a single field of view, a typical next step is to compile and analyze a collection of extracted traces across multiple imaged animals. The traces exhibit variability due to both methodological variability (e.g., variability inherent in imaging equipment) and biological variability (e.g., variability inherent in the levels of fluorescent-protein expression across neurons of the same type). These "extra" sources of variability can obscure the changes in neural activity that we wish to extract and analyze here. Consequently, a neuron's calcium trace, measured across multiple animals, can exhibit differences in overall intensity that require correction to obtain valid comparisons across animals. As a simple example, many neuron classes are composed of a symmetric left and right pair that often show identical calcium activity. With most imaging equipment, when the left neuron is near the lens, the corresponding right neuron is far away, leading to a false differential reading of brightness. Thus, even within a single animal, symmetric neurons can require corrections to be comparable.

The commonly used technique of converting neural traces to $\Delta F / F_{0}$ aims to correct these issues in mismatched fluorescence intensity profiles but is often insufficient (see Results section below and Figure 2). One way to further normalize time-series data is through $\mathrm{z}$-scoring the signal such that the mean and variance across time is zero and one, respectively. However, in practice, simply mean-shifting to zero often misrepresents the neuron's baseline signal. Similarly, scaling to unit variance will scale unresponsive and responsive neurons to the same magnitude, thus inflating instead of suppressing measurement noise in unresponsive cells.

A method that employs a more robust view of the distribution of neural signal would provide a more accurate normalization. Here we generalize the concept of $\mathrm{z}$-scoring timeseries by first observing that z-scoring is a linear transform that matches the histogram of the time series to a standard Gaussian distribution with zero mean and unit variance. We then cast histogram normalization in a way such that the transformation is constrained to be a linear transform that minimizes distance to the distribution as a whole, leading to more robust results compared to z-scoring, which restricts attention to two non-robust summary statistics of the histogram (the mean and variance). Lastly, we provide a strategy for normal- 
izing a population of time series data by transforming to the medoid of these time series (i.e., the time series which is on average closest to all the others in the population). Empirically, the resulting approach preserves signal while reducing variability across the population.

2.8.1 Quantile regression: Let $C^{i} \in \mathbb{R}^{T}$ denote the time series of a neuron in the $i$ th animal over $T$ time steps. Suppose we want to match the neural time-series of the $i$ th animal to ${ }_{411}$ the $j$ th animal using a linear transform. One possible strategy ${ }_{412}$ to match two time-series signals to one another is to match ${ }_{413}$ their baselines and match their peaks. This corresponds to ${ }_{414}$ transforming the minimum and the maximum of one time se- ${ }_{415}$ ries such that they match the minimum and maximum of the ${ }_{410}$ other time series. This is equivalent to matching their minimal and maximal quantiles through a transformation term involving scaling and shifting.

We can generalize this procedure with more quantiles to yield a transformation estimate that is more robust to noise. Matching multiple quantiles using a linear transformation term can be represented by the following linear model:

$$
F_{\boldsymbol{C}^{j}}^{-1}(a)=F_{\boldsymbol{C}^{i}}^{-1}(a) \nu+\nu_{0}+\epsilon
$$

where $F^{-1}$ denotes the inverse cumulative distribution function and $\nu, \nu_{0}$ denote the scaling and magnitude shift of the time-series, respectively, and $\epsilon$ represents an error term. This model posits that each time series signal consists of a baseline ${ }_{417}$ and several peaks which can be represented as quantiles of histograms that require matching; baselines and peaks of the same neuron, across different animals, should roughly have similar values.

We can then estimate $\nu$ and $\nu_{0}$ by solving the following least squares problem:

$$
W_{2, L}\left(\boldsymbol{C}^{i}, \boldsymbol{C}^{j}\right)=\min _{\nu, \nu_{0}} \int_{0}^{1}\left\|F_{\boldsymbol{C}^{j}}^{-1}(a)-F_{\boldsymbol{C}^{i}}^{-1}(a) \nu-\nu_{0}\right\|_{2}^{2} d a .
$$

The arg-min of Eq. (11) yields the linear estimates $\nu, \nu_{0}$ that can be used to transform the time series $C^{i}$ to match the time series $\boldsymbol{C}^{j}$, where $\hat{\boldsymbol{C}}^{i, j}=\boldsymbol{C}^{i} \nu^{*}+\nu_{0}^{*}$. We term this regression model, quantile regression $(\mathrm{QR})$, since the predictors and responses are quantiles of time-series data. If only two quantiles are used i.e. the bottom and topmost quantiles, this procedure is equivalent to matching the minimum/maximum of the two time-series.

Optimizing Eq. (11) yields the transformation that best matches the histogram of the ith time series $C^{i}$ with that of the jth time series $C^{j}$. The residual discrepancy between ${ }_{418}$ the transformed ith time series $\hat{C}^{i, j}$ and the $j$ th time series ${ }_{419}$ $C^{j}$ can be thought of a distance between these time series.420 In fact, the minimum of Eq. (11) is a linear approximation ${ }_{421}$ of a bona fide distance metric, termed the Wasserstein met-422 ric [Peyré et al., 2019], that is a distance between probability ${ }_{423}$ distributions.

Using this notion of proximity between time series, if we ${ }_{425}$ have a population of $N$ samples $C^{1}, \ldots, C^{N}$, the strategy ${ }_{426}$ for normalizing the time series we advocate here is to compute pairwise Wasserstein distance approximations between all time series and choose the medoid time series to normalize to:

$$
\boldsymbol{C}^{0} \leftarrow \arg \min _{\boldsymbol{C}^{\ell}} \sum_{i=1}^{N} W_{2, L}\left(\boldsymbol{C}^{\ell}, \boldsymbol{C}^{i}\right)
$$

In other words, we can find the best fit of each time series through quantile regression to all other time series, and set as a reference the time series that has the minimal average distance to all the other time series. Once the reference is set, all the samples are transformed to match the reference quantiles using Eq. (11). See Figure 2 for an illustration.

Lastly, if the time series all capture non-negative signal (as is often encountered in calcium imaging) the regression in Eq. (11) can be constrained to be non-negative to ensure the transformed time series maintains its positivity. This yields the non-negative linear estimate of the Wasserstein metric. We term this variant of the quantile regression model as nonnegative quantile regression $(\mathrm{NQR})$ :

$$
W_{2, N}\left(\boldsymbol{C}^{i}, \boldsymbol{C}^{j}\right)=\min _{\nu, \nu_{0} \geq 0} \int_{0}^{1}\left\|F_{\boldsymbol{C}^{j}}^{-1}(a)-F_{\boldsymbol{C}^{i}}^{-1}(a) \nu-\nu_{0}\right\|_{2}^{2} d a
$$

\subsection{Evaluation metrics}

To evaluate the performance of the proposed method as well as the compared methods, we focus on several metrics that shed light both on the signal demixing capabilities of the methods as well as their ability to track objects in time. Namely we focus on two major metrics: trajectory correlation, which measures the ability of the deformation model to keep track of the observed motion, and signal correlation, which measures the demixing performance by comparing the correlation of demixed signal intensities relative to the ground truth. Specifically, these metrics can be expressed as

\section{Trajectory correlation:}

$$
\rho(\hat{\boldsymbol{\beta}}, \boldsymbol{\beta})=\frac{\sum_{i, j, t}\left(\hat{\beta}_{t}^{i j}-\overline{\hat{\beta}}\right)\left(\beta_{t}^{i j}-\bar{\beta}\right)}{\sqrt{\sum_{i, j, t}\left(\hat{\beta}_{t}^{i j}-\overline{\hat{\beta}}\right)^{2}} \sqrt{\sum_{i, j, t}\left(\beta_{t}^{i j}-\bar{\beta}\right)^{2}}}
$$

Signal correlation:

$$
\rho(\hat{\boldsymbol{C}}, \boldsymbol{C})=\frac{\sum_{k t}\left(\hat{C}_{k t}-\overline{\hat{C}}\right)\left(C_{k t}-\bar{C}\right)}{\sqrt{\sum_{k t}\left(\hat{C}_{k t}-\overline{\hat{C}}\right)^{2}} \sqrt{\sum_{k t}\left(C_{k t}-\bar{C}\right)^{2}}} .
$$

The above metrics are applicable when the ground truth motion trajectories and the signal coefficients are known. To evaluate the performance of the methods using unsupervised registration heuristics, we focus on the correlation of registered frames to the average frame (after registration). Heuristically, this measure has been demonstrated to be an effective indicator of successful registration [Pnevmatikakis and Giovannucci, 2017]. Furthermore, in the real data experiments, we also evaluate the average 

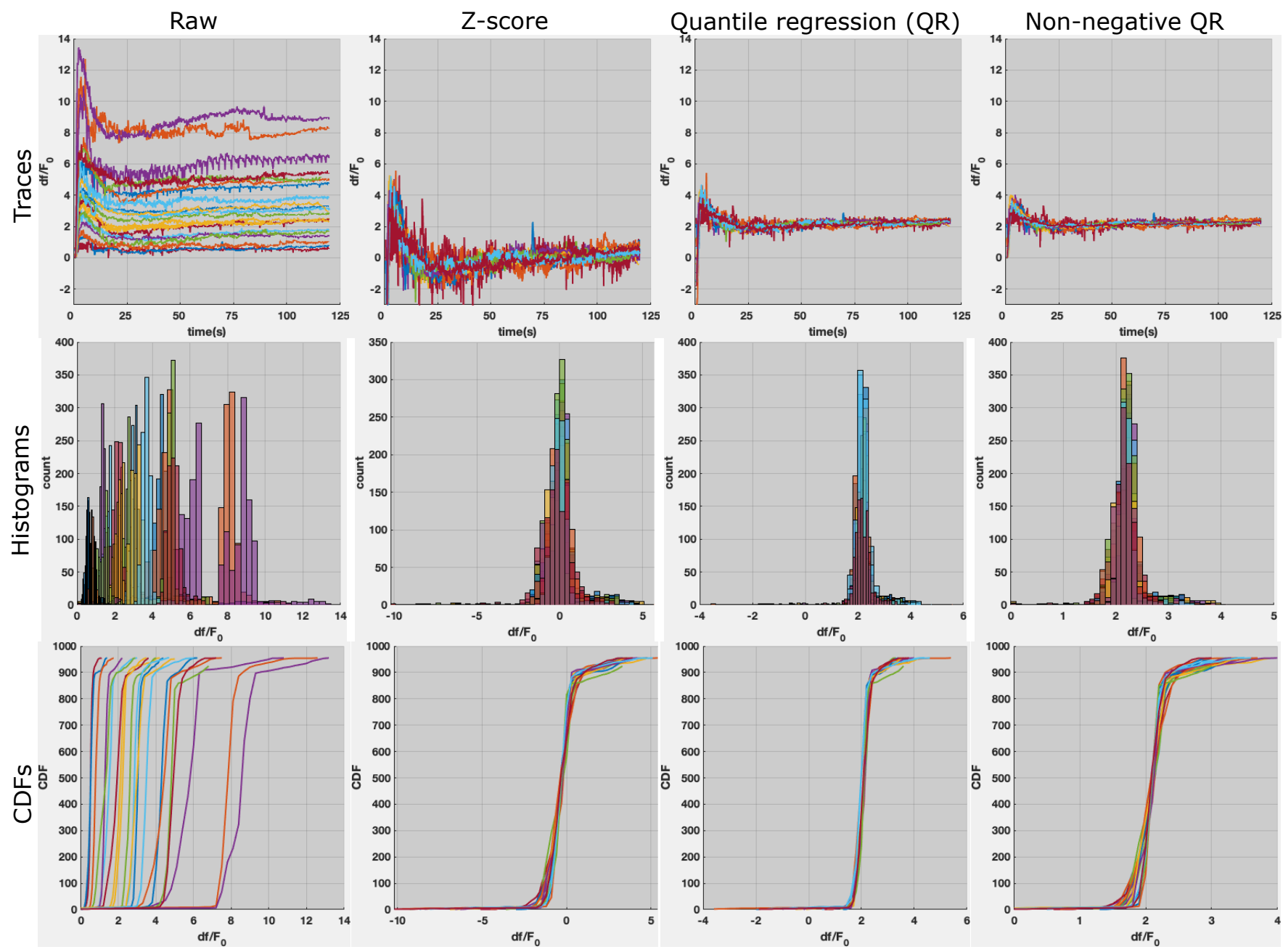

Fig. 2. A demonstration of quantile regression for the tail neuron LUAL in C. elegans, across 21 animals. Different colors indicate different animals. First column: The raw traces superimposed exhibit variability in intensity profiles due to imaging and biological differences (top). The histograms and cumulative distribution functions (CDFs) of the time-series signals display the differing distributions representing these traces (middle and bottom). Second column: Z-scored traces exhibit tighter grouping than raw traces (top) further shown in their CDFs (bottom). However, these z-scored traces are shifted towards zero mean (top) which is misrepresentative of the signal magnitude and also exhibit significant remaining variability. Third and fourth columns: After quantile regression (QR) and non-negative quantile regression (NQR) to the medoid of the traces, we see that the normalized traces retain their shape (top), exhibiting even tighter grouping than after z-scoring. In comparison with the $\mathrm{z}$-Scored traces, both QR and NQR preserve the median signal magnitude, $\Delta F / F_{0}=\sim 2$ (top and middle) with smaller tails in their histogram (middle), implying a better fit across the population of animals.

spread of cell locations before and after registration. This is 445 computed by taking the average distance of the cells to the average cell location. Similar to the frame correlation measure, this metric allows us to diagnose whether certain cells are ${ }^{447}$ registered better than others. While the average correlation ${ }^{448}$ of frames is a high-level measure of registration performance, ${ }^{449}$ the measure of cellular spread is a localized metric, indicat- ${ }^{450}$ ing whether certain regions of the volume are registered bet- ${ }^{451}$ ter than others. In other words, the former metric measures ${ }_{452}^{452}$ global sharpness while the latter measures local sharpness.

The added benefit of the latter two evaluation metrics ${ }^{456}$ is that since they do not require any ground truth, they ${ }^{457}$ can be used for hyperparameter selection; i.e., we can se-- ${ }^{458}$ lect regularization parameters that yield the sharpest regis- ${ }^{459}$ tration results. Furthermore, we can use the sharpness cri- ${ }^{460}$ teria to evaluate the goodness of fit for different deforma-461 tion models such as quadratic polynomials (as used here), b-462 splines [Rueckert et al., 1999], or higher order polynomials. ${ }_{463}$

\subsection{Compared methods}

We argue in this paper that jointly optimizing for deformable registration and time-series signal extraction has the potential to improve the quality of both the registration and signal extraction. Therefore, we compare the registration performance of dNMF against the state-of-theart method for calcium video motion registration, named Normcorre [Pnevmatikakis and Giovannucci, 2017]. Normcorre does not explicitly model the presence of independent signal carrying units in the FOV and instead performs piecewise-rigid transformations on overlapping sub-blocks of the volume using a fast fourier transform based technique [Guizar-Sicairos et al., 2008]. Furthermore, Normcorre uses a normalized cross-correlation registration loss function that is less prone to intensity variations across timeframes.

Next, we also evaluate the signal extraction performance of dNMF against two standard routines in calcium imaging. First, we compare against region of in- 
terest (ROI) tracking and pixel averaging within the ROI 519 [Venkatachalam et al., 2016a]. This method tracks the posi-520 tions of cells across time and extracts signal by taking the 521 average pixel intensity value in a pre-defined radial region 522 around the tracking marker. We also compare against the rou-523 tine of performing motion correction first and then signal ex-524 traction through NMF [Pnevmatikakis et al., 2016]. To repli-525 cate this routine in our experiments, we motion correct using 526 Normcorre and then use the Gaussian cell shape parametriza-527 tion version of NMF that is described in section 2. We use ${ }_{528}$ this variant of NMF rather than non-parametric variants such ${ }_{529}$ as CNMF [Pnevmatikakis et al., 2016] to bring the compari-530 son against dNMF to an equal footing since dNMF already ${ }_{531}$ uses this parametrization that tends to model nuclear shapes 532 well.

\subsection{Implementation details}

All the optimization codes are implemented in Python ${ }^{536}$ 3.7 .3 using the autograd tool and the PyTorch $1.5^{537}$ package. We used the Adam optimizer with learning rate ${ }^{538}$ 0.001 for the simulations and 0.00001 for the worm exper- ${ }^{539}$ iments. Large learning rates lead to jumps in the tracks ${ }^{540}$ and lower quality traces, while small learning rates need ${ }^{541}$ more iterations to converge. The experiments are run on a Lenovo X1 laptop with Microsoft Windows operating system ${ }^{542}$ using 64 GB RAM and Intel(R) Core(TM) i7-8850H CPU @ ${ }_{543}$ 2.60GHz, 2592 Mhz, 6 Core(s), 12 Logical Processor(s). We 544 further implemented a sequential optimizer for the demixing 545 of an online stream of videos where each batch of data con-546 sisting of a few time frames of the video is processed with ${ }_{547}$ parameters initialized using the previous batch. In addition, 548 to improve the memory and time efficiency of our algorithms 549 we also introduced a stochastic variant of dNMF, where in 550 each iteration, to compute the loss and its gradient, we ran-551 domly subsample the pixels both in the spatial and temporal 552 domains and update the parameters based on those samples. ${ }_{553}$

\subsection{C. elegans video description}

Videos of calcium activity in $C$. elegans were cap- ${ }^{556}$ tured via a spinning-disk confocal microscope with res- ${ }^{557}$ olution $(\mathrm{x}, \mathrm{y}, \mathrm{z})=(0.27,0.27,1.5)$ microns. Whole-brain cal-558 cium activity was measured using the fluorescent sensor ${ }^{559}$ GCaMP6s in animals expressing a stereotyped fluorescent ${ }^{560}$ color map that permitted class-type identification of every ${ }^{561}$ neuron in the worm's brain (NeuroPAL strain OH16230) ${ }^{562}$ [Yemini et al., 2019]. Each video was 4 minutes long and was acquired at approximately $4 \mathrm{~Hz}$. Worms were par- ${ }_{563}$ alytically immobilized (using tertramisole) in a microfluidic chip capable of delivering chemosensory stimuli (salt ${ }^{564}$ and two odors) [Chronis et al., 2007, Si et al., 2019]. This ${ }_{565}$ setup allows for the controlled delivery of multiple solu-566 ble stimuli to the animal with high-temporal precision. See ${ }_{567}$ [Yemini et al., 2019] for full experimental details.

Despite paralytic immobilization, we still observed some ${ }_{569}$ motion of the worm within the chip, primarily over small dis-570 tances of several microns and over slow, multi-second time 571 scales. Some of this motion was driven by the animal, while 572 some was the result of the animal drifting passively due to minute pressure differences in the chip. This motion was strongest in the tail, which, due to its taper, was not well secured by the channel walls of the microfluidic chip. Despite the smaller scale of this motion (as compared to freelymoving behavior such as crawling), motion artifacts could strongly confound traces, particularly in the head of the animal where the neurons are very tightly packed. Thus, these motion artifacts required algorithmic correction.

Each dataset from this collection is a video in the form of a 4D tensor $W \times H \times D \times T$ (approximately $256 \times 128 \times$ $21 \times 960)$ where the value of the tensor at $(x, y, z, t)$ corresponds to the activity of a neuron located near the point $(x, y, z)$ at time $t$. To extract the neural activity from the videos we first reformat the data into a $d \times T$ matrix where $d=W H D$ that is called the data matrix $\boldsymbol{Y}$. We then run the Gaussian dNMF with cell centers initialized using the cell locations in the initial frame, determined using the semiautomated methods described in [Yemini et al., 2019]. Since the cells are approximately spherical in this video we used a fixed spherical covariance matrix for all the cells with squared root diagonal entries equal to $0.57 \mu \mathrm{m}$ (roughly a third of the minimal diameter of adult worm neurons).

\subsection{Pre-processing steps}

Neuron centers were first tracked using a local image registration approach throughout the time series, using the approach in [Venkatachalam et al., 2016a]. After identifying each neuron center in the first frame, every subsequent frame was registered to this first frame. The registration was performed on $x-, y$-, and $z$ - maximum-intensity projections of a small volume around the neuron center using the imregister function in MATLAB. The volume was chosen to be small enough that nonrigid deformations could generally be neglected, so we used a rigid registration model (translation and rotation only). Because motion is continuous between frames, the initial guess for the transformation was taken to be the calculated transformation from the previous time frame.

We use the initial trajectories of the neurons to initialize our motion parameters $\boldsymbol{\beta}_{t}$ by solving $\boldsymbol{\beta}_{t}=$ $\arg \min _{\beta}\left\|\boldsymbol{\beta} \psi\left(P_{1}\right)-P_{t}\right\|_{2}^{2}$ where $P_{t}$ contains the locations of neurons in time $t$ tracked using local image registration techniques. For computational efficiency, we also mask out pixels that are outside of the circles with radii $3 \mu \mathrm{m}$ from the location of all neurons in all time points.

\section{Results}

\subsection{Simulation experiments}

To evaluate the effectiveness of our algorithm in capturing motion and demixing time-series traces, we simulated the trajectory of 10 neurons, with a time-specific trace assigned to each (Fig. 3A-B). The signal for each neuron is modeled as a binary vector with length $T$ and probability $p$ of observing a unit spike, convolved with a decaying exponential kernel. Each trajectory was generated using quadratic transformations of the point cloud in its previous time point, starting 
bioBxiv preprint doi: https://doi.org/10.1101/2020.07.07.192120; this version posted July 8, 2020. The copyright holder for this preprint (which

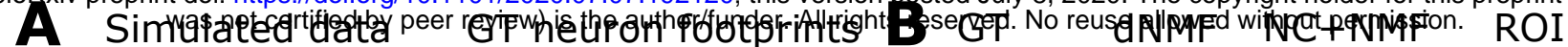
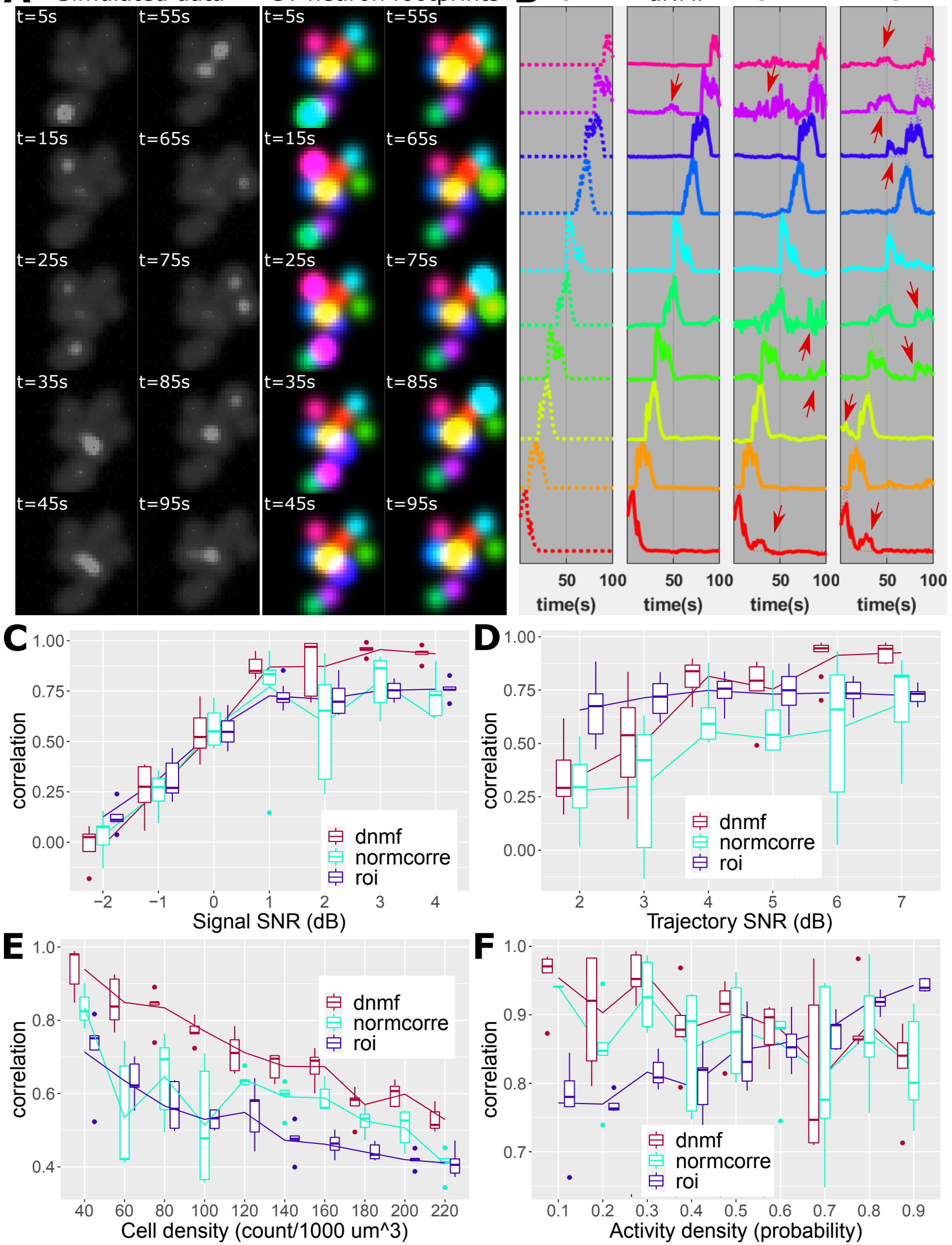

Fig. 3. Demixing calcium signals in simulated videos. A: Neurons are generated as Gaussian shapes and undergo motion and simulated calcium activity in a 100-second long video. Static snapshots of the video are shown (left) and spatial footprints for each cell are assigned unique colors with intensities proportional to calcium activity (right). Note that the spatial footprints of cells are also in motion, tracking the position of the cells. B: The ground truth calcium activity for each cell (left) is compared with the neural activity extracted using dNMF (second column), Normcorre [Pnevmatikakis and Giovannucci, 2017]+NMF (third column) and ROI tracking and pixel averaging (fourth column). dNMF recovers the ground truth signal well whereas Normcorre+NMF and ROI methods yield significantly more mixed signals (indicated by red arrows) due to the proximity of the cells and the tendency of the spatial footprints of mobile cells to overlap. C: The correlation of the recovered signal to the ground truth signal as a function of the image signal-to-noise ratio (SNR). D: The correlation of the recovered cell movement trajectories to the ground truth trajectories as a function of trajectory SNR. E: The correlation of the recovered signals to the ground truth as a function of the density of independent objects in the FOV. F: The correlation of the recovered signals to the ground truth as a function of the density of signaling events (simulating neural excitation) exhibited by the cells. Note that we provided ROI tracking here with access to the ground truth cell centers at all times (explaining why ROI averaging correlation values remain high even in the limit of very high activity density); nonetheless, even with artificially perfect tracking accuracy, mixing of nearby signals remains a significant issue. See MOVIE LINK for further details. 

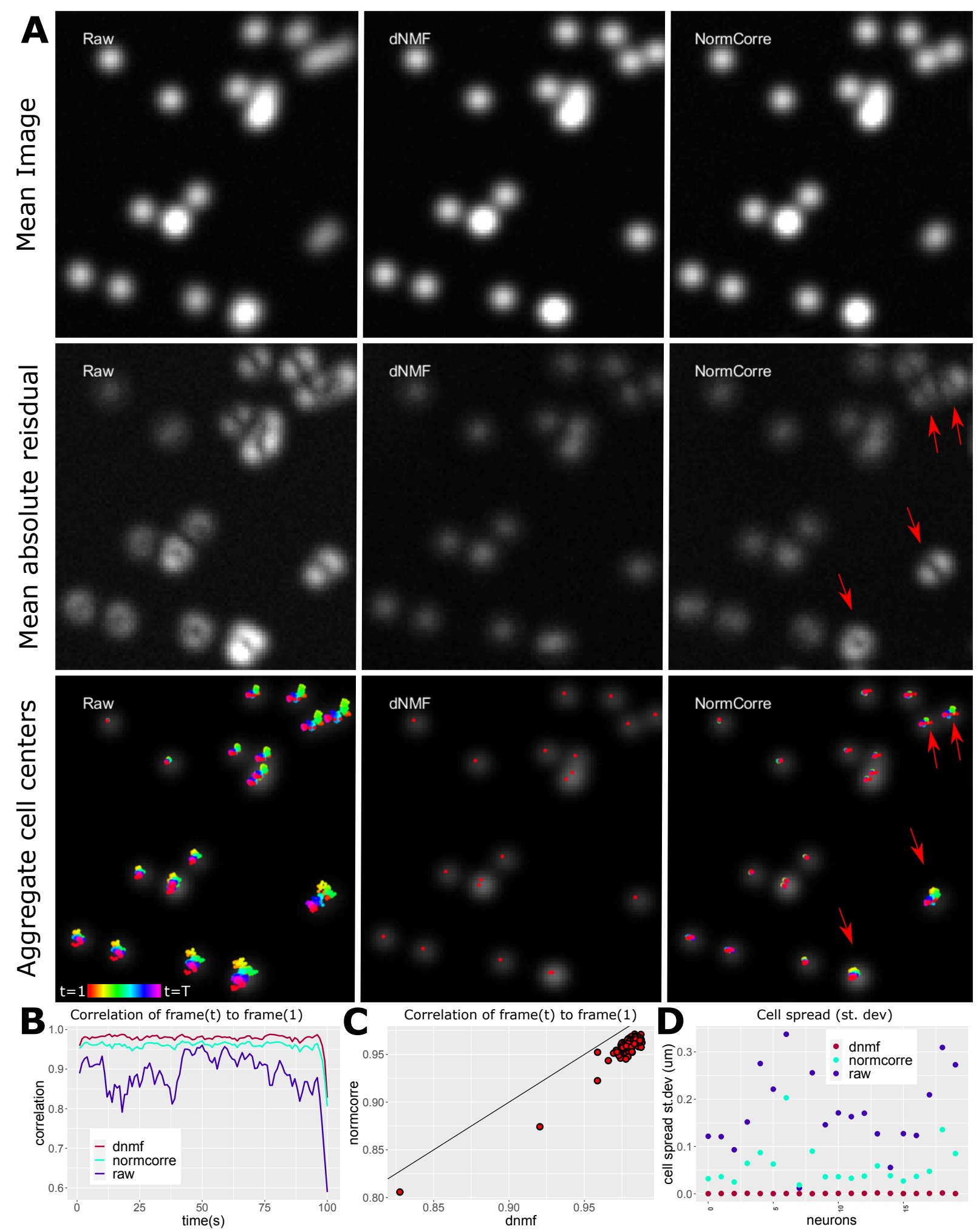

Fig. 4. Simulated data registration results. A: Top row: The mean video frame prior to registration (left), after dNMF based registration (middle), and after Normcorre [Pnevmatikakis and Giovannucci, 2017] registration. Middle Row: Mean of the absolute value of the video frames subtracted from the first frame prior to registration (left), after dNMF based registration (middle) and after Normcorre (right). If registration is perfect, this image will look like a weighted sum of Gaussian shapes, one for each cell (corresponding to the cell dimming and brightening, but remaining in place); imperfect registrations are indicated by "spreading" or "doubling" of the cell shapes, as indicated by red arrows. Bottom row: The locations of the cells across time (colors denote different times) superimposed on the first frame prior to registration (left), after dNMF based registration (middle) and after Normcorre (right). Red arrows indicate cells with imperfect registration, with significant remaining movement of the cells across frames. B: The correlation of the video frames to the mean video frame before registration (blue), after dNMF based registration (red), and after Normcorre (cyan); higher values indicate better performance here. C: The correlation of individual registered frames to the mean video frame after dNMF registration ( $\mathrm{x}$-values) and Normcorre ( $\mathrm{y}$-values). The straight line indicates $x=y$; points below this line indicate the higher correlation of dNMF registered frames to the mean frame. D: The spread of the cell position centers, relative to their average. in the unregistered video (blue), after dNMF-based registration (red), and after Normcorre (cyan). A lower standard deviation for cell spread indicates better performance for local registration of cell shapes. See MOVIE LINK for further details. 
A
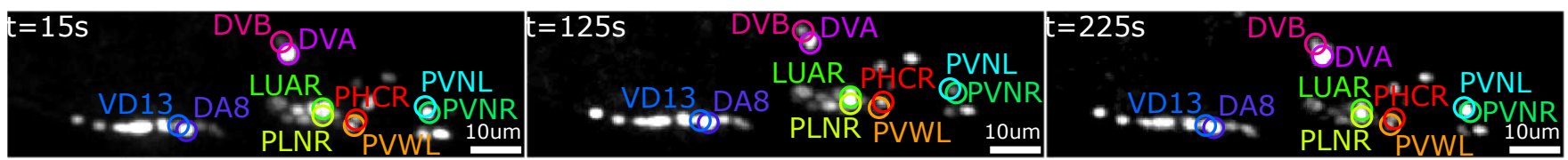

\section{B dNMF}
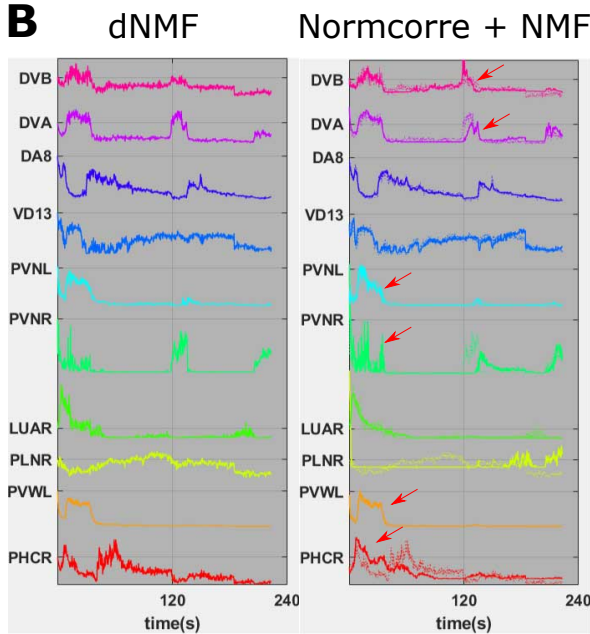

D

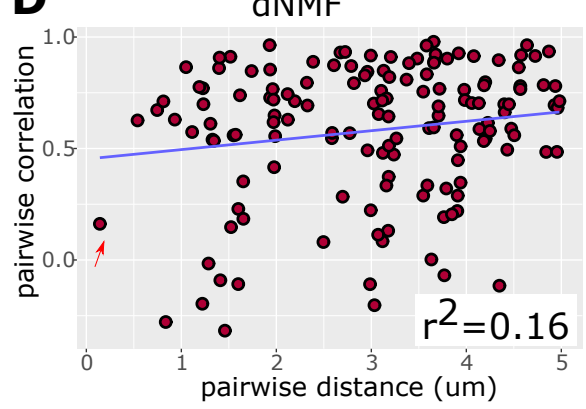

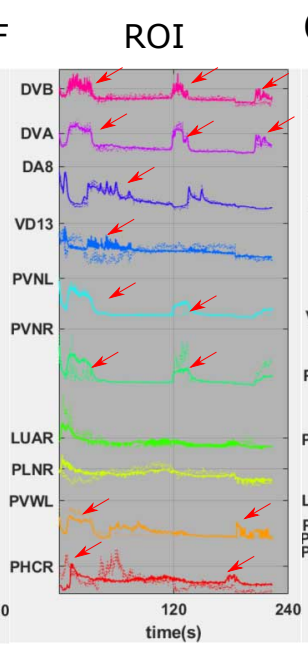

C dNMF
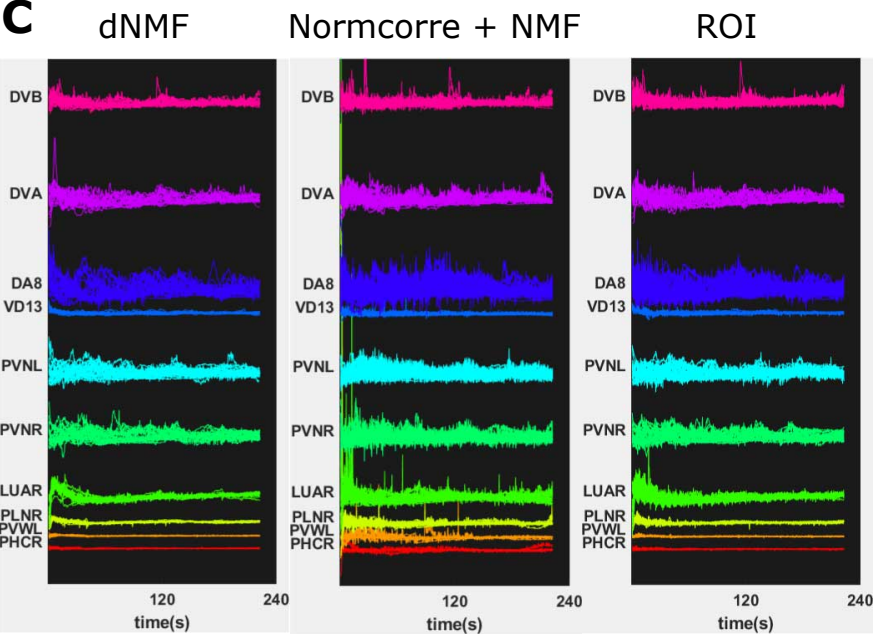

Normcorre + NMF

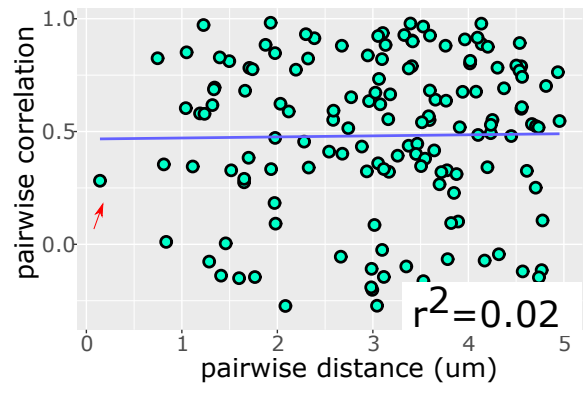

Fig. 5. Demixing neural calcium signal in semi-immobilized $C$. elegans videos. A: Three static, z-axis maximum projected frames from a representative 4-minute long video of GCaMP6s neural activity. We focus on the signal from five pairs of spatially-neighboring neurons in the tail: DVA/DVB, PVNR/PVNL, PVWL/PHCR, PLNR/LUAR, and VD13/DA8. B: Calcium signals extracted by dNMF (left), Normcorre [Pnevmatikakis and Giovannucci, 2017] + NMF (middle), and ROI tracking and averaging (right). dNMF extracts uncoupled signals that demonstrate independent neural activity. The selected cells were chosen such that the signal recovered by ROI averaging is inconsistent with dNMF (quantified by having correlation smaller than 0.4). Normcorre + NMF partially mixes signals between both PHCR/PVWL and PVNL/PVNR around the 30-second mark and DVB/DVA around the 120-second mark (red arrows), and loses nearly all signal from PLNR, due to motion exhibited by the semi-immobilized animal. ROI averaging produces completely correlated signal (red arrows) between all of the labeled neurons, and loses most of the signal from LUAR and PLNR, due to overlap in their spatial footprints. C: Calcium activity traces, of the labeled tail neurons, extracted from a population of 21 worms. The unique colors label traces from the same neurons, across different animals. Here, the dNMF traces are tightly grouped, exhibiting minimal variability between animals. Normcorre+NMF traces exhibit mixed-signal and mistracked neurons. ROI traces exhibit wider variability than dNMF, due to mixed signals and, potentially, noise common to ROI averaging. D: Pairwise neuron distances versus pairwise correlation of neural signals for all three methods. Note that signal mixing tends to occur when the signal sources are close to one another, necessitating techniques such as NMF to disentangle independent signals. For this reason, dNMF is well suited to demix spatially-close neuron pairs. Normcorre+NMF experiences mixing effects due to motion for which it fails to account (seen in the supplementary movie linked below). ROI averaging does mix traces and thus shows increasingly correlated signals between neuron pairs as they get nearer to each other (indicated by the red arrow). See MOVIE LINK for further details.

from a random initial point cloud. (Note that the composition 586 of many such quadratic mappings is non-quadratic, and there-587 fore the generative model here does not perfectly match the 588 model dNMF uses to fit the data, where a quadratic transfor-589 mation maps the spatial components $A$ to match the observed 590 data at each frame; nonetheless, despite this model mismatch, 591 dNMF achieves accurate results here, as discussed below.) ${ }_{592}$ The trajectory of each neuron was then convolved with a fixed 593 3D Gaussian filter that represents the shape of that neuron and then multiplied with the time course assigned to that neuron. ${ }^{594}$ The simulated video is the result of the superposition of these ${ }^{595}$ moving Gaussian functions.

We compare the performance of dNMF, Norm-598
corre+NMF, and ROI pixel averaging in a variety of confounding scenarios using the metrics defined in section 9 . In all simulation experiments, the ROI averaging method is provided with the ground truth cell positions - i.e., we examine the accuracy of this method under the (unrealistically optimistic) assumption that neurons are tracked perfectly, to evaluate the demixing performance of ROI signal extraction without the additional confound of tracking performance.

In Fig. 3C-F, we explore the performance limits of dNMF, Normcorre+NMF, and ROI pixel averaging as a function of imaging noise and motion variability. Signal SNR is defined by the peak-to-trough difference between the neural activity signals during times of activity. Trajectory SNR is quanti- 


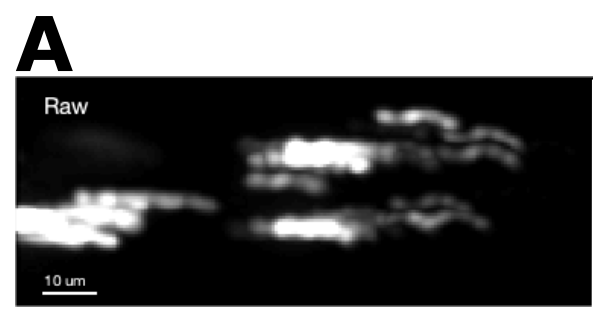

Mean Image

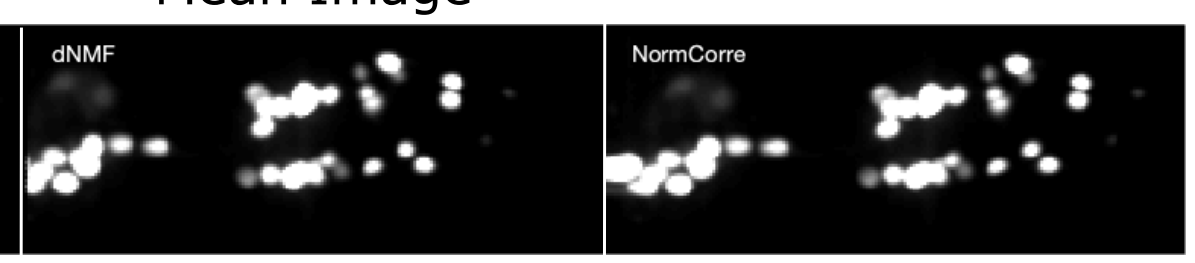

Mean absolute residual
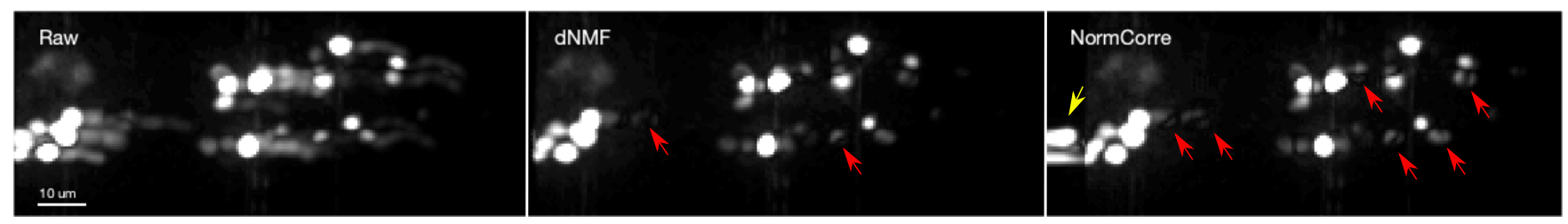

\section{Aggregate cell centers}
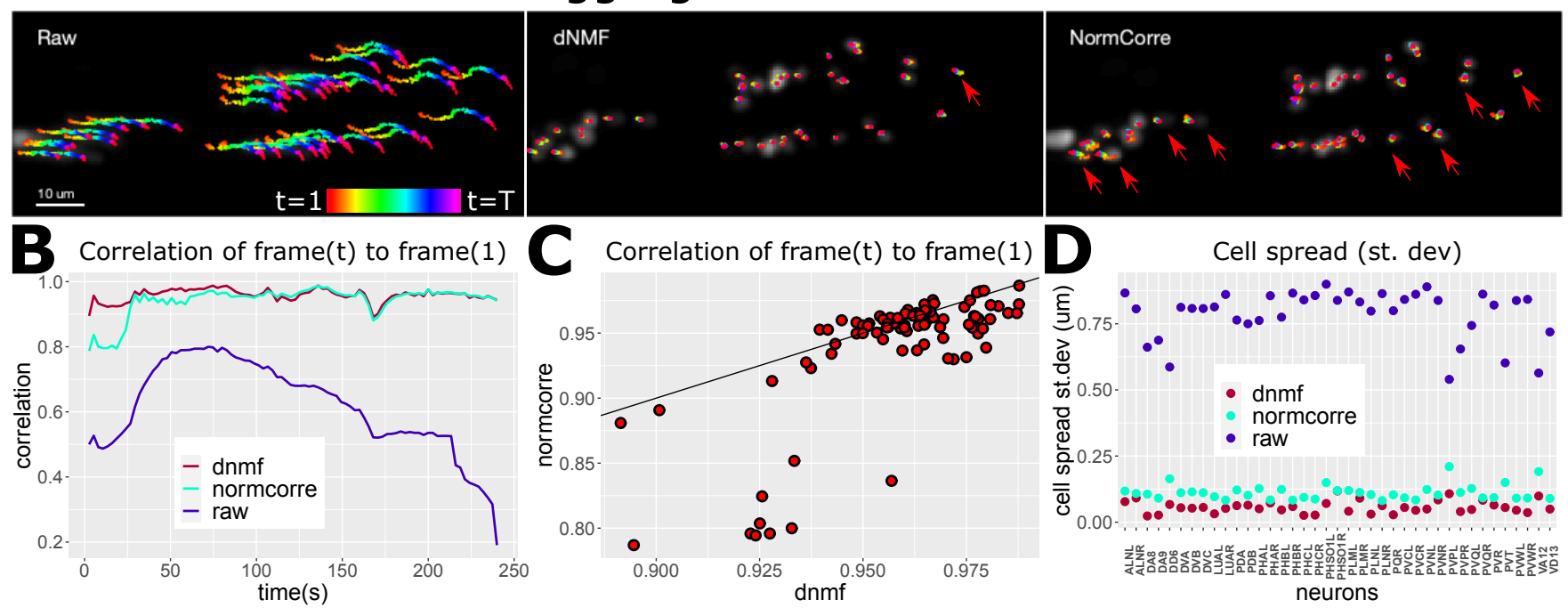

Fig. 6. C. elegans neural activity video registration results. A: Top row: The mean video frame prior to registration (left), after dNMF based registration (middle), and after Normcorre (right). Overall conventions are similar to Fig. 4. Middle row: The mean, of the absolute value, of the difference between the first video frame (prior to registration) and subsequent video frames (left). We show these results for dNMF-base registration (middle) and Normcorre (right). Distorted toroidal shapes (indicated by red arrows) denote the superposition of mismatched spatial footprints, indicating a misestimation of deformation. The yellow arrow indicates Normcorre's boundary pixel extrapolation, which introduces blocky artifacts. Bottom row: The positions of cells over time superimposed on the first video frame (left), after dNMF-based registration (middle), and after Normcorre (right). Tighter grouping of cell centers indicates a good correction of motion. Spread groupings of cells indicate poor registration (indicated by red arrows). B: Correlation of the video frames to the mean frame, across time, for the unregistered video (blue), after dNMF-based registration (red), and after Normcorre (cyan). dNMF slightly outperforms Normcorre here. C: Correlation of the individual video frames, to the mean video frame, after registering with dNMF ( $\mathrm{x}$-values) and after Normcorre (y-values). The solid line denotes $x=y$. Points below this line (which indicate a higher correlation of registered frames to the average frame) represent better performance for dNMF, and points above this line represent better performance for Normcorre. D: The spread of the cell position centers, relative to their average. in the unregistered video (blue), after dNMF-based registration (red), and after Normcorre (cyan). Again, a lower standard deviation for cell spread indicates better performance for local registration of cell shapes. See MOVIE LINK for further details.

fied by how well the cells adhere to the motion of all other ${ }_{614}$ cells; high trajectory SNR indicates all cells move in unison, ${ }_{615}$ resembling a deformable medium, and low trajectory SNR in-616 dicates each cell is moving like independent particles. Math-617 ematically, this is proportional to the log ratio of the variance ${ }_{618}$ of the average location of the cells versus the variance of the ${ }_{619}$ time differences of these locations. It can be seen in Fig. $3 \mathrm{D}_{620}$ that dNMF is robust to noise but ultimately may introduce ${ }_{621}$ errors to demixing and trajectory tracking if the signal and ${ }_{622}$ trajectory SNR (Fig. 3D) are too low. Normcorre+NMF does ${ }_{623}$ relatively worse than dNMF as a function of signal SNR and ${ }_{624}$ trajectory SNR. ROI pixel averaging has the poorest signal ${ }_{625}$ recovery performance of the three compared methods as $\mathrm{a}_{626}$ function of signal SNR. (Note that ROI pixel averaging en- ${ }_{627}$ joys a constant trajectory estimation rate in Fig. 3D, since it ${ }_{628}$ has access to ground truth cell locations, as discussed above.)

Next, we evaluated the signal extraction performance as a function of the cell density in the FOV. Increased cell density indicates an increased superpositioning of independent signals and therefore a higher degree of signal mixing. dNMF demixing performance degrades linearly as the density of independent objects within the FOV increases (Fig. 3E) but enjoys higher rates of recovery than both Normcorre+NMF and ROI pixel averaging.

Lastly, we observe that the density of signaling events changes the demixing performance for the three compared methods. In particular, low signal densities (simulating weak excitation) make it harder to track individual cells, which may be dim and therefore hard to detect and track in many frames. dNMF does not suffer in the low signal density regime since 

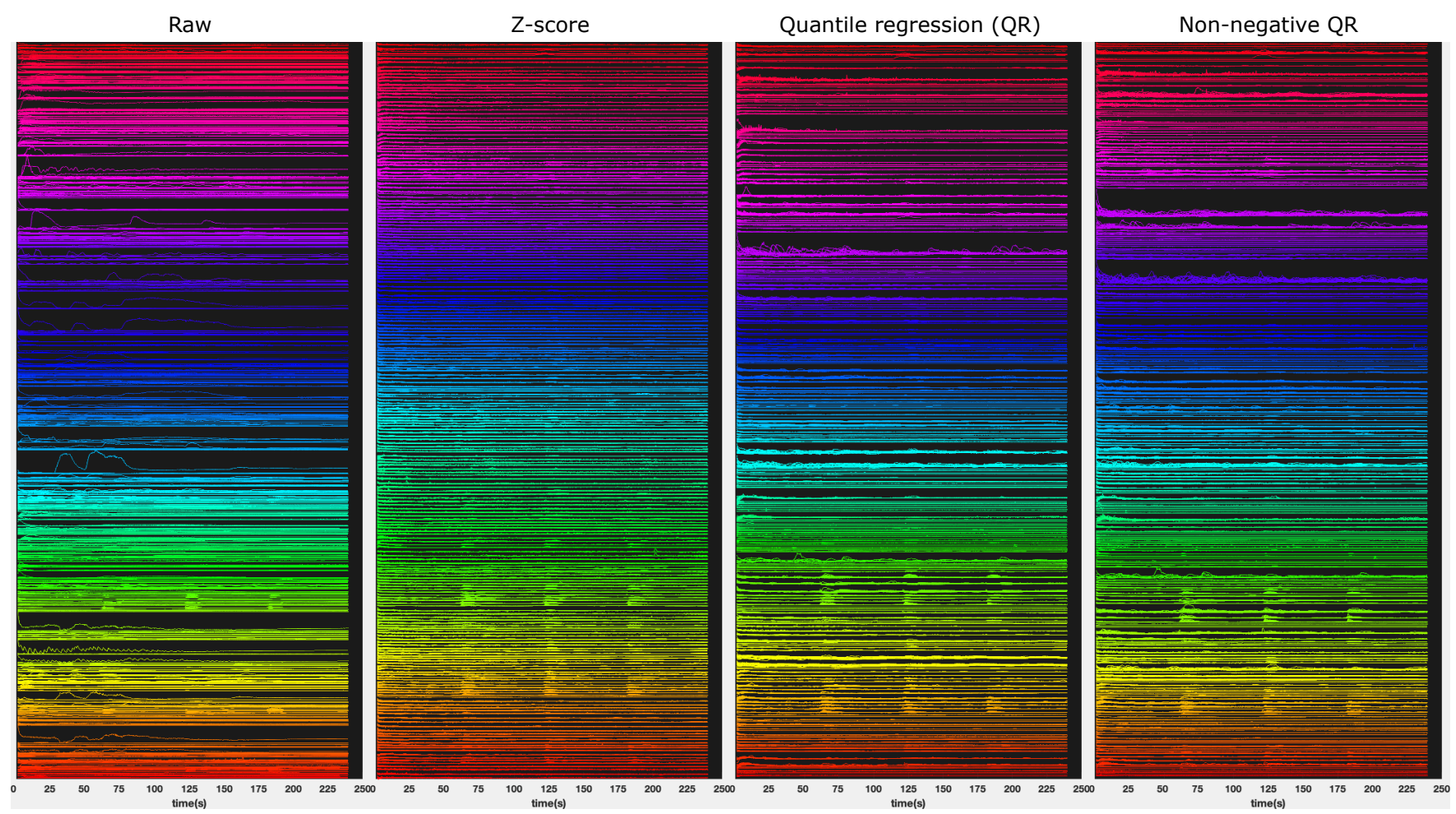

Fig. 7. dNMF was used to motion correct, extract, and demix calcium traces of $C$. elegans neurons from 21 animal heads and 21 animal tails. We demonstrate four strategies for superimposing multi-animal traces for different neuron types. First column: Raw traces superimposed, colors indicate different neuron types. Within the same color, different traces indicate different animals. (Y-axis: neuron types), (X-axis) time (s). Second column: Z-scored neuron time series. Third column: Quantile regression (QR) normalized time series. Fourth column: Non-negative quantile regression (NQR). Z-scores use only two summary statistics (mean and variance) for normalization. Z-score scaling to unit variance is strongly influenced by any large-magnitude fluctuations in the signal. Consequently, in a mixture of responsive and unresponsive traces from the same neuron, across multiple animals, z-scored traces with a response will be scaled to match their unresponsive counterparts, thus muting signal in these traces. This is exhibited by the compressed appearance of the z-scored traces in the second column. In contrast, quantile regression uses a more robust and rich set of summary statistics to determine an appropriate scaling. As such responsive and unresponsive neural traces retain appropriate differential scales. This is exhibited by the quantile regression methods shown in third and fourth columns which show better preservation of neuron responses when compared to their z-scored equivalents. Additionally, the z-score translates to zero mean and thus can misrepresent the signal baseline. In contrast, both QR methods preserve the correct signal baseline and, when appropriate, the NQR method can be used to further maintain non-negativity of signals (see Fig. 2).

it combines information across all visible cells to update the 652 tracking model, and this helps to track dim cells as well.

In Fig. 4, we qualitatively demonstrate the reg-654 istration performance of dNMF versus Normcorre 655 [Pnevmatikakis and Giovannucci, 2017]. We see that 656 the average frame, after registering with dNMF, is sharper 657 than the non-registered average frame, with better-localized 658 and less-variable cell center locations. In comparison, Norm-659 corre yields a higher spread of cells, even after registration, 660 which may lead to erroneous signal recovery. Both of 661 these global and local sharpness metrics are quantified in 662 Fig. 4C-D.

\subsection{Demonstration of demixing in real C. elegans data ${ }^{665}$} In the simulated data analyzed above, dNMF exhibits supe ${ }_{-667}^{666}$ rior registration performance due to its ability to decouple ${ }^{667}$ the intensity signal from the motion of objects. Conversely, coupling registration with signal extraction enables dNMF to ${ }_{670}$ capture the neural signal and demix it from nearby cells more accurately.

We extend this demonstration further with a real data 672 example. The worm's tail contains several ganglia, with 673 densely-packed neurons, whose spatial footprints often over-674 lap due to insufficient spatial resolution. Additionally, even 675 neurons in separate ganglia can end up in sufficient proximity, due to microfluidic confinement or other imaging-setup induced deformations, such that their spatial footprints overlap. The spatial overlap represents a significant challenge, both for tracking individual neurons and demixing their signal. Figure 5 shows an example of the difficulty present when tracking and demixing neural activity signals from animals with spatially overlapping neural footprints in their recorded images. In this example, ROI tracking loses most of the signal from the LUAR and PLNR neurons and further mixes signals between the DA8/VD13, DVA/DVB, PHCR/PVWL, and PVNL/PVNR neurons. Normcorre+NMF performs better but loses nearly all signals from PLNR while also still mixing signals between the DVA/DVB, PHCR/PVWL, and PVNL/PVNR neurons. In comparison, dNMF recovers strong, independent signals from all ten neurons. Thus dNMF can track and differentiate signals from neurons, even within areas containing multiple spatially-overlapping neural footprints where other comparable algorithms fail.

Additionally, in figure 5, we quantify the demixing performance by computing the pairwise correlation of nearby neurons as a function of the distance between these neurons. Signal mixing is expected to occur when the spatial footprints of nearby neurons, blurred by the point spread function and/or 
insufficient spatial resolution, overlap with another. There-73 fore, one heuristic to determine how well demixing was per-732 formed is the correlation of pairwise distances of neurons 733 to the pairwise correlations of their activity. Indeed, both ${ }_{734}$ matrix factorization methods, dNMF and Normcorre+NMF, 735 yield uncorrelated trends between neuron pair distance and 736 the correlation of their respective trace activity. On the other ${ }^{737}$ hand, simple ROI averaging tends to visibly mix signals in 738 closely-neighboring neurons, resulting in unrealistically high 739 correlation values near 1 for the closest neighbors.

\subsection{Worm registration}

After optimizing dNMF, we can obtain registered videos of ${ }^{743}$ worms to evaluate performance and compare against Norm- ${ }^{744}$ corre (Figure 6A). Similar to simulated data, we can once ${ }^{745}$ again observe that the mean video frame after registering ${ }^{746}$ with dNMF is sharper when compared to both the raw av- ${ }^{747}$ erage frame and this average after applying Normcorre. Fur ${ }^{748}$ thermore, the mean of the absolute difference between video ${ }^{749}$ frames and the first frame shows that the dNMF registration ${ }^{750}$ has fewer distorted toroidal shapes than Normcorre, indicat- ${ }^{75}$ ing better registration of cell shapes. Lastly, we can also ${ }^{752}$ see that after registering with dNMF the cell centers have $\mathrm{a}^{753}$ tighter grouping than Normcorre; this is another indication of ${ }^{754}$ better registration performance.

Figure 6B-D evaluates these observations quantitatively. ${ }^{750}$ The subfigures $\mathrm{B}$ and $\mathrm{C}$ indicate that the frames registered ${ }^{757}$ via dNMF tend to have a higher correlation to the mean reg- ${ }^{758}$ istered frame than Normcorre, which indicates the quality of ${ }^{759}$ registration. Furthermore, the dNMF and Normcorre registra- ${ }^{760}$ tion results diverge most in the initial frames, where the majority of deformable motions are observed. Since Normcorre 761 is a piecewise rigid registration technique, its deformation model may be misspecified to capture such motions, whereas ${ }_{76}^{76}$ the dNMF motion model is more accurate. Figure 6D demonstrates that the cell grouping after dNMF is indeed tighter ${ }^{764}$ than Normcorre.

\subsection{Population study of C. elegans}

Using the neural traces extracted with dNMF (converted ${ }_{769}$ to $\left.\Delta F / F_{0}\right)$, we demonstrate the time-series histogram-770 normalization technique of quantile regression $(\mathrm{QR})$, show 771 its non-negative regression variant (NQR), and compare these 772 with z-score normalization. The time-series data we used is 773 the brain-wide neural GCaMP6s intensity extracted from 21774 worm heads (up to 189 neurons in each head) and 21 worm 775 tails (up to 42 neurons in each tail). In these animals, neu-776 rons with the same identity often exhibited very different in- 777 tensity distributions across individual animals. In the course 778 of a time series, neuron intensities change to reflect the un-779 derlying activities but, given a sufficiently long recording, af- -780 ter proper alignment, the probability density function $(\mathrm{PDF})_{781}$ should be roughly equivalent for neurons of the same class ${ }_{782}$ type.

Differences in the intensity PDFs of neurons with identi-784 cal class types are due to variability in imaging conditions, 785 anisotropy due to random animal orientations, and biological 786 variability in fluorescence expression. To properly compare one animal to another, class-specific neural intensity distributions must be corrected so that they match each other appropriately (e.g., all LUA neurons should exhibit similar PDFs); otherwise, this variability will distort population representations of the signal. In figure 2, we explore these population representations of signal by focusing on a single neuron, LUAL, to compare raw, z-scored, QR, and NQR normalized neural traces. Although the LUAL neurons should preserve similar PDFs, instead they exhibit high variability in both their signal magnitude and baseline activity in their traces, histograms, and cumulative distribution functions (CDFs). Zscoring partially corrects this variability but retains long tails in the PDFs (histograms), while shifting them to zero mean, which is far less than the median signal observed in the raw traces (a median $\Delta F / F_{0}$ of approximately 2 ). In comparison, QR and NQR reduce LUAL neural variability substantially, when compared to z-scoring. Moreover, both QR and NQR preserve the median exhibited by the raw traces and, thereby, retain a better approximation of the neural baseline, whereas $\mathrm{z}$-scoring distorts this baseline.

In figure 7, we extend our demonstration to all head and tail neurons. In this broader representation of neural activity, one can see that the raw traces and even the z-scored traces distort the neural signal, exhibiting a flat appearance with outliers flanking this flattened signal. In contrast, the QR and NQR traces exhibit strong signals without obvious outliers. Thus both QR and NQR can correct variability in neural intensities to help compare signals from neurons with identical types, recorded from a population of animals.

\section{Discussion}

In this paper, we considered the problem of extracting and demixing calcium signals from microscopy videos of $C$. elegans. We developed an extension of NMF, with a nonlinear motion model applied to the spatial cellular footprints, to deform the static image of these cells, modeling the worm's posture at each time frame. We provided two different parameterizations for the spatial footprints (Gaussian functions or a non-parametric model) and described regularizations that can help in finding smooth trajectories and signals. We further showed that our method outperforms state-of-the-art models that use a two-step process for motion stabilization/tracking and signal extraction. Finally, we demonstrated the effectiveness of our model by extracting calcium signals from videos of semi-immobilized C. elegans.

The dNMF framework we introduce is a generalization of standard non-negative matrix factorization techniques that are commonly used to demix, deconvolve, and denoise neural signals in calcium imaging [Pnevmatikakis et al., 2016]. To tackle the added problem of deforming tissues and mobile animals, we have additionally adopted techniques from the image-registration community to quantify motion in the factorization model. The overall framework is modular and may be further generalized to incorporate different image fidelity loss functions, different regularizers, and alternative deformation parametrizations. Currently, we use a Eu- 
clidean loss to assess the goodness-of-fit of the factorization 838 model compared to the observations. Alternative choices for the loss function include normalized local cross correla- ${ }_{840}$ tion [Sotiras et al., 2013]. Similarly, we found it sufficient to ${ }^{840}$ use a quadratic polynomial basis for quantifying the motions ${ }^{841}$ exhibited by semi-immobilized $C$. elegans worms. However, ${ }^{842}$ different animal models or freely-moving worms may neces- ${ }_{843}$ sitate the use of higher-order deformation models such as ${ }_{844}$ free-form b-splines [Rueckert et al., 1999].

One common obstacle, after extracting neural traces from 846 a population of animals, is that imaging and biological variability can lead to high variability in the intensity values of ${ }^{847}$ traces measured from the same neuron, across multiple ani- ${ }^{848}$ mals. To address this, we have provided a time-series nor- ${ }^{849}$ malization technique called quantile regression $(\mathrm{QR})$. This ${ }^{850}$ technique bears similarities to $\mathrm{z}$-scoring but it is more ro- ${ }_{851}$ bust to outliers in the time series, does not artificially enforce ${ }_{852}$ a uniform signal variance over all cells, and yields a more ${ }_{853}$ consistent and tighter normalization across a population of ${ }_{854}$ neural traces. Moreover, QR can optionally enforce the non- ${ }_{855}$ negativity of the signal that is transformed and normalized (as opposed to z-scoring, which introduces negative values). 856

Finally, in this work we focused on nuclear-localized ${ }^{857}$ calcium imaging in semi-immobilized C. elegans. We ${ }^{858}$ believe that a similar approach will be useful with ${ }^{859}$ other indicators [Chen et al., 2020] and in other prepara- ${ }_{860}$ tions, e.g. larval zebrafish [Vanwalleghem et al., 2018], ${ }_{861}$ Drosophila [Schaffer et al., 2020], and Hydra ${ }_{862}$ [Szymanski and Yuste, 2019]; see, in particular, the recent ${ }_{863}$ preprint by [Lagache et al., 2020], who develop improved tracking methods that may nicely complement the dNMF 864 approach. We look forward to exploring these directions 865 further in future work.

\section{Software}

The software is implemented in Python us- ${ }^{870}$ ing the Pytorch library and is available at ${ }_{871}$ https://github.com/amin-nejat/dNMF.

\section{Acknowledgements}

The authors thank Ruoxi Sun, Conor McGrory, Shreya Sax- ${ }^{876}$ ena, and Scott Linderman for the helpful discussions and ${ }^{877}$ suggestions. We also acknowledge the following fund- ${ }^{878}$ ing sources. Paninski Lab: NSF NeuroNex Award DBI- ${ }^{879}$ 1707398, The Gatsby Charitable Foundation, NIBIB R01 ${ }^{880}$ EB22913, DMS 1912194, Simons Foundation Collaboration ${ }_{881}$ on the Global Brain. Eviatar Yemini: Howard Hughes Med- ${ }_{882}$ ical Institute, NIH (5T32DK7328-37, 5T32DK007328-35, ${ }_{883}^{882}$ 5T32MH015174-38, and 5T32MH015174-37), Samuel Lab: ${ }_{884}^{883}$ NIH (1R01NS113119-01) and NSF (IOS-1452593). Albert Lin: NSF Physics of Living Systems Graduate Student Re-885 search Network (1806818). Venkatachalam Lab: Burroughs 886 Wellcome Fund Career Award at the Scientific Interface. $\quad{ }_{887}$

\section{References}

[Ahrens et al., 2013] Ahrens, M. B., Orger, M. B., Robson, D. N., Li, J. M., and Keller, P. J. (2013). Whole-brain functional imaging at cellular resolution using light-sheet microscopy. Nature Methods, 10(5):413-420.

[Andilla and Hamprecht, 2013] Andilla, F. D. and Hamprecht, F. A. (2013). Learning multi-level sparse representations. In Advances in Neural Information Processing Systems, pages 818-826.

[Andilla and Hamprecht, 2014] Andilla, F. D. and Hamprecht, F. A. (2014). Sparse space-time deconvolution for calcium image analysis. In Advances in neural information processing systems, pages 64-72.

[Barbera et al., 2016] Barbera, G., Liang, B., Zhang, L., Gerfen, C. R., Culurciello, E., Chen, R., Li, Y., and Lin, D.T. (2016). Spatially compact neural clusters in the dorsal striatum encode locomotion relevant information. Neuron, 92(1):202-213.

[Chen et al., 2020] Chen, Y., Jang, H., Spratt, P. W., Kosar, S., Taylor, D. E., Essner, R. A., Bai, L., Leib, D. E., Kuo, T.-W., Lin, Y.-C., et al. (2020). Soma-targeted imaging of neural circuits by ribosome tethering. Neuron .

[Chronis et al., 2007] Chronis, N., Zimmer, M., and Bargmann, C. I. (2007). Microfluidics for in vivo imaging of neuronal and behavioral activity in Caenorhabditis elegans. Nature Methods, 4(9):727-731.

[Dombeck et al., 2007] Dombeck, D. A., Khabbaz, A. N., Collman, F., Adelman, T. L., and Tank, D. W. (2007). Imaging large-scale neural activity with cellular resolution in awake, mobile mice. Neuron, 56(1):43-57.

[Dubbs et al., 2016] Dubbs, A., Guevara, J., and Yuste, R. (2016). moco: Fast motion correction for calcium imaging. Frontiers in neuroinformatics, 10:6.

[Flusberg et al., 2008] Flusberg, B. A., Nimmerjahn, A., Cocker, E. D., Mukamel, E. A., Barretto, R. P., Ko, T. H., Burns, L. D., Jung, J. C., and Schnitzer, M. J. (2008). High-speed, miniaturized fluorescence microscopy in freely moving mice. Nature methods, 5(11):935-938.

[Girard et al., 2007] Girard, L. R., Fiedler, T. J., Harris, T. W., Carvalho, F., Antoshechkin, I., Han, M., Sternberg, P. W., Stein, L. D., and Chalfie, M. (2007). Wormbook: the online review of caenorhabditis elegans biology. $\mathrm{Nu}$ cleic acids research, 35(suppl_1):D472-D475.

[Göbel et al., 2007] Göbel, W., Kampa, B. M., and Helmchen, F. (2007). Imaging cellular network dynamics in three dimensions using fast $3 \mathrm{~d}$ laser scanning. Nature methods, 4(1):73-79.

[Guizar-Sicairos et al., 2008] Guizar-Sicairos, M., Thurman, S. T., and Fienup, J. R. (2008). Efficient subpixel image registration algorithms. Optics letters, 33(2):156-158. 
[Haeffele et al., 2014] Haeffele, B., Young, E., and Vidal, R.938 (2014). Structured low-rank matrix factorization: Opti-эзя mality, algorithm, and applications to image processing.940 In International conference on machine learning, pages 941 2007-2015.

[Hirose et al., 2017] Hirose, O., Kawaguchi, S., Tokunaga,943 T., Toyoshima, Y., Teramoto, T., Kuge, S., Ishihara, T.,944 Iino, Y., and Yoshida, R. (2017). Spf-celltracker: Track-945 ing multiple cells with strongly-correlated moves using a 946 spatial particle filter. IEEE/ACM transactions on computational biology and bioinformatics, 15(6):1822-1831.

[Hofer et al., 2011] Hofer, S. B., Ko, H., Pichler, B., Vogel-949 stein, J., Ros, H., Zeng, H., Lein, E., Lesica, N. A., and 950 Mrsic-Flogel, T. D. (2011). Differential connectivity and response dynamics of excitatory and inhibitory neurons in ${ }^{951}$ visual cortex. Nature neuroscience, 14(8):1045.

[Kato et al., 2015] Kato, S., Kaplan, H. S., Schrödel, T.,954 Skora, S., Lindsay, T. H., Yemini, E., Lockery, S., and 955 Zimmer, M. (2015). Global brain dynamics embed the 956 motor command sequence of caenorhabditis elegans. Cell, 163(3):656-669.

[Kerlin et al., 2010] Kerlin, A. M., Andermann, M. L., Bere-959 zovskii, V. K., and Reid, R. C. (2010). Broadly tuned re-960 sponse properties of diverse inhibitory neuron subtypes in mouse visual cortex. Neuron, 67(5):858-871.

[Kerr et al., 2005] Kerr, J. N., Greenberg, D., and Helmchen, 963 F. (2005). Imaging input and output of neocortical net-964 works in vivo. Proceedings of the National Academy of 965 Sciences, 102(39):14063-14068.

[Klein et al., 2009] Klein, A., Andersson, J., Ardekani,967 B. A., Ashburner, J., Avants, B., Chiang, M.-C., Chris-968 tensen, G. E., Collins, D. L., Gee, J., Hellier, P., et al.969 (2009). Evaluation of 14 nonlinear deformation algorithms applied to human brain mri registration. Neuroim- 970 age, 46(3):786-802.

[Lagache et al., 2020] Lagache, T., Hanson, A., Fairhall, A.,973 and Yuste, R. (2020). Robust single neuron tracking of 974 calcium imaging in behaving hydra. bioRxiv.

[Larsch et al.,2013] Larsch, J., Ventimiglia, D., Bargman, C. I., and Albrecht, D. R. (2013). High-throughput 977 imaging of neuronal activity in caenorhabditis ele-978 gans. Proceedings of the National Academy of Sciences, 979 110(45):E4266-E4273.

[Lee and Seung, 1999] Lee, D. D. and Seung, H. S. (1999).981 Learning the parts of objects by non-negative matrix fac-982 torization. Nature, 401(6755):788-791.

[Lee and Seung, 2001] Lee, D. D. and Seung, H. S. (2001). Algorithms for non-negative matrix factorization. In $A d-985$ vances in neural information processing systems, pages 986 $556-562$.
[Mann et al., 2017] Mann, K., Gallen, C. L., and Clandinin, T. R. (2017). Whole-brain calcium imaging reveals an intrinsic functional network in drosophila. Current biology : $C B, 27(15): 2389-2396 . e 4$. 28756955[pmid].

[Maruyama et al., 2014] Maruyama, R., Maeda, K., Moroda, H., Kato, I., Inoue, M., Miyakawa, H., and Aonishi, T. (2014). Detecting cells using non-negative matrix factorization on calcium imaging data. Neural Networks, 55:1119.

[Mukamel et al., 2009] Mukamel, E. A., Nimmerjahn, A., and Schnitzer, M. J. (2009). Automated analysis of cellular signals from large-scale calcium imaging data. Neuron, 63(6):747-760.

[Nguyen et al., 2016] Nguyen, J. P., Shipley, F. B., Linder, A. N., Plummer, G. S., Liu, M., Setru, S. U., Shaevitz, J. W., and Leifer, A. M. (2016). Whole-brain calcium imaging with cellular resolution in freely behaving caenorhabditis elegans. Proceedings of the National Academy of Sciences, 113(8):E1074-E1081.

[Niell and Smith, 2005] Niell, C. M. and Smith, S. J. (2005). Functional imaging reveals rapid development of visual response properties in the zebrafish tectum. Neuron, 45(6):941-951.

[O'grady and Pearlmutter, 2006] O'grady, P. D. and Pearlmutter, B. A. (2006). Convolutive non-negative matrix factorisation with a sparseness constraint. In 2006 16th IEEE Signal Processing Society Workshop on Machine Learning for Signal Processing, pages 427-432. IEEE.

[Paatero and Tapper, 1994] Paatero, P. and Tapper, U. (1994). Positive matrix factorization: A non-negative factor model with optimal utilization of error estimates of data values. Environmetrics, 5(2):111-126.

[Pachitariu et al., 2013] Pachitariu, M., Packer, A. M., Pettit, N., Dalgleish, H., Hausser, M., and Sahani, M. (2013). Extracting regions of interest from biological images with convolutional sparse block coding. In Advances in neural information processing systems, pages 1745-1753.

[Pachitariu et al., 2017] Pachitariu, M., Stringer, C., Dipoppa, M., Schröder, S., Rossi, L. F., Dalgleish, H., Carandini, M., and Harris, K. D. (2017). Suite2p: beyond 10,000 neurons with standard two-photon microscopy. Biorxiv.

[Peng et al., 2012] Peng, Y., Ganesh, A., Wright, J., Xu, W., and Ma, Y. (2012). Rasl: Robust alignment by sparse and low-rank decomposition for linearly correlated images. IEEE transactions on pattern analysis and machine intelligence, 34(11):2233-2246.

[Peyré et al., 2019] Peyré, G., Cuturi, M., et al. (2019). Computational optimal transport. Foundations and Trends ${ }^{\circledR}$ in Machine Learning, 11(5-6):355-607. 
[Pnevmatikakis and Giovannucci, 2017] Pnevmatikakis, 1039 E. A. and Giovannucci, A. (2017). Normcorre: An $\mathbf{n}_{1040}$ online algorithm for piecewise rigid motion correction of 1041 calcium imaging data. Journal of neuroscience methods, $; 042$ 291:83-94.

[Pnevmatikakis et al., 2016] Pnevmatikakis, E. A., Soudry ${ }_{1044}^{1043}$ D., Gao, Y., Machado, T. A., Merel, J., Pfau, D., Reardon, T., Mu, Y., Lacefield, C., Yang, W., et al. (2016). Simulta $\overline{1}_{046}$ neous denoising, deconvolution, and demixing of calcium imaging data. Neuron, 89(2):285-299.

[Poole et al., 2015] Poole, B., Grosenick, L., Broxton, M. ${ }_{1049}^{1048}$ Deisseroth, K., and Ganguli, S. (2015). Robust nonrigid alignment of volumetric calcium imaging data. COSYNE.[Google Scholar].

[Prevedel et al., 2014] Prevedel, R., Yoon, Y.-G., Hoffmann, ${ }^{1052}$ M., Pak, N., Wetzstein, G., Kato, S., Schrödel, T., Raskar, ${ }^{1053}$ R., Zimmer, M., Boyden, E. S., et al. (2014). Simultaneous whole-animal $3 \mathrm{~d}$ imaging of neuronal activity using light ${ }^{1054}$ field microscopy. Nature methods, 11(7):727-730.

[Reidl et al., 2007] Reidl, J., Starke, J., Omer, D. B., Grin ${ }^{1057}$ vald, A., and Spors, H. (2007). Independent component analysis of high-resolution imaging data identifies distinct ${ }^{1058}$ functional domains. Neuroimage, 34(1):94-108. 1059

[Rueckert et al., 1999] Rueckert, D., Sonoda, L. I., Hayes, ${ }_{0061}$ C., Hill, D. L., Leach, M. O., and Hawkes, D. J. (1999) ${ }_{1062}$ Nonrigid registration using free-form deformations: application to breast $\mathrm{mr}$ images. IEEE transactions on medical1063 imaging, 18(8):712-721.

[Saxena et al., 2019] Saxena, S., Kinsella, I., Musall, S. ${ }_{9066}^{1065}$ Kim, S. H., Meszaros, J., Thibodeaux, D. N., Kim, C., Cunningham, J., Hillman, E., Churchland, A., and Panin ${ }_{7067}$ ski, L. (2019). Localized semi-nonnegative matrix fac fac6 $_{7067}$ torization (locanmf) of widefield calcium imaging data ${ }_{4069}$ bioRxiv.

[Schaffer et al., 2020] Schaffer, E., Mishra, N., Li, W., et al ${ }_{1072}^{1071}$ (2020). flygenvectors: large-scale dynamics of internal ${ }_{1073}^{1072}$ and behavioral statesin a small animal. COSYNE, (III-19).

[Schrödel et al., 2013] Schrödel, T., Prevedel, R., Aumayr, ${ }^{1074}$ K., Zimmer, M., and Vaziri, A. (2013). Brain-wide $3 \mathrm{~d}^{1075}$ imaging of neuronal activity in caenorhabditis elegans ${ }^{1076}$ with sculpted light. Nature methods, 10(10):1013.

[Si et al., 2019] Si, G., Kanwal, J. K., Hu, Y., Tabone, C. J.,1079 Baron, J., Berck, M., Vignoud, G., and Samuel, A. D. (2019). Structured Odorant Response Patterns across $a^{1080}$ Complete Olfactory Receptor Neuron Population. Neuron ${ }^{1081}$ 101(5):950-962.e7.

[Siegel et al., 2007] Siegel, R. M., Duann, J.-R., Jung, T.-P. and Sejnowski, T. (2007). Spatiotemporal dynamics of the functional architecture for gain fields in inferior parietal ${ }_{1085}$ lobule of behaving monkey. Cerebral cortex, 17(2):378-086 390.
[Smaragdis, 2006] Smaragdis, P. (2006). Convolutive speech bases and their application to supervised speech separation. IEEE Transactions on Audio, Speech, and Language Processing, 15(1):1-12.

[Sotiras et al., 2013] Sotiras, A., Davatzikos, C., and Paragios, N. (2013). Deformable medical image registration: A survey. IEEE transactions on medical imaging, 32(7):1153-1190.

[Stetter et al., 2001] Stetter, M., Greve, H., Galizia, C. G., and Obermayer, K. (2001). Analysis of calcium imaging signals from the honeybee brain by nonlinear models. Neuroimage, 13(1):119-128.

[Szymanski and Yuste, 2019] Szymanski, J. R. and Yuste, R. (2019). Mapping the whole-body muscle activity of hydra vulgaris. Current Biology, 29(11):1807-1817.

[Taslaman and Nilsson, 2012] Taslaman, L. and Nilsson, B. (2012). A framework for regularized non-negative matrix factorization, with application to the analysis of gene expression data. PLOS ONE, 7(11):1-7.

[Tian et al., 2009] Tian, L., Hires, S. A., Mao, T., Huber, D., Chiappe, M. E., Chalasani, S. H., Petreanu, L., Akerboom, J., McKinney, S. A., Schreiter, E. R., et al. (2009). Imaging neural activity in worms, flies and mice with improved gcamp calcium indicators. Nature methods, 6(12):875.

[Vanwalleghem et al., 2018] Vanwalleghem, G. C., Ahrens, M. B., and Scott, E. K. (2018). Integrative whole-brain neuroscience in larval zebrafish. Current opinion in neurobiology, 50:136-145.

[Venkatachalam et al., 2016a] Venkatachalam, V., Ji, N., Wang, X., Clark, C., Mitchell, J. K., Klein, M., Tabone, C. J., Florman, J., Ji, H., Greenwood, J., Chisholm, A. D., Srinivasan, J., Alkema, M., Zhen, M., and Samuel, A. D. T. (2016a). Pan-neuronal imaging in roaming Caenorhabditis elegans. Proceedings of the National Academy of Sciences of the United States of America, 113(8):E1082-8.

[Venkatachalam et al., 2016b] Venkatachalam, V., Ji, N., Wang, X., Clark, C., Mitchell, J. K., Klein, M., Tabone, C. J., Florman, J., Ji, H., Greenwood, J., et al. (2016b). Pan-neuronal imaging in roaming caenorhabditis elegans. Proceedings of the National Academy of Sciences, 113(8):E1082-E1088.

[Voleti et al., 2019] Voleti, V., Patel, K. B., Li, W., Campos, C. P., Bharadwaj, S., Yu, H., Ford, C., Casper, M. J., Yan, R. W., Liang, W., et al. (2019). Real-time volumetric microscopy of in vivo dynamics and large-scale samples with scape 2.0. Nature methods, 16(10):1054-1062.

[Yang and Yuste, 2017] Yang, W. and Yuste, R. (2017). In vivo imaging of neural activity. Nature methods, 14(4):349. 
[Yemini et al., 2019] Yemini, E., Lin, A., Nejatbakhsh, A., Varol, E., Sun, R., Mena, G. E., Samuel, A. D., Paninski, L., Venkatachalam, V., and Hobert, O. (2019). Neuropal: A neuronal polychromatic atlas of landmarks for wholebrain imaging in c. elegans. BioRxiv, page 676312.

[Zeng et al., 2016] Zeng, K., Erus, G., Sotiras, A., Shinohara, R. T., and Davatzikos, C. (2016). Abnormality detection via iterative deformable registration and basis-pursuit decomposition. IEEE transactions on medical imaging, 35(8):1937-1951.

[Zhou et al., 2018] Zhou, P., Resendez, S. L., RodriguezRomaguera, J., Jimenez, J. C., Neufeld, S. Q., Giovannucci, A., Friedrich, J., Pnevmatikakis, E. A., Stuber, G. D., Hen, R., et al. (2018). Efficient and accurate extraction of in vivo calcium signals from microendoscopic video data. Elife, $7:$ e28728. 


\section{Video captions}

\subsection{Simulation demixing video (VIDEO LINK)}

Simulated data demixing results. Left block: In the top left ${ }^{1162}$ we see a max projection of the raw video to be demixed. This ${ }^{116}$ video involves 10 neurons in motion with time-varying cal- ${ }^{1164}$ cium activity. (For better visibility, note that we use a differ- ${ }^{116}$ ent simulated dataset here than in Figure 3.) In the middle we ${ }^{116}$ see the reconstruction of this video using the moving spatial ${ }^{116}$ components extracted with dNMF; each extracted cell is ran- ${ }^{1160}$ domly assigned a unique color. On the top right, we see the ${ }^{1169}$ residual of the raw video minus the reconstruction. The next row shows a selection of cells zoomed and centered on the motion tracking position inferred by dNMF, followed by the corresponding spatial component with intensity proportional ${ }^{11}$ to the extracted activity level. Note that the neuron centered ${ }^{117}$ panels exhibit little motion, showing that each zoomed cell ${ }^{115}$ has been successfully tracked. The following row shows the same cells but with the ground truth signal intensities, and ${ }^{117}$ then the localized residual. Finally, the bottom row shows the extracted signals (in the color assigned to each cell) $\mathrm{vs}^{11790}$ the ground truth (black).

Right block: This block has the same conventions as the left block but with Normcorre [Pnevmatikakis and Giovannucci, 2017] motion ${ }^{1182}$ corrected video instead of dNMF. The raw data in this ${ }^{1183}$ panel is motion corrected through Normcorre and thus the ${ }^{1184}$ reconstruction, residual and the zoom panels are all in the ${ }^{1185}$ context of the motion corrected video.

As noted above, upon zooming in on the motion corrected ${ }^{1187}$ patches around each cell, we can see that dNMF provides a ${ }^{118}$ good stabilization of the centered cell in each zoomed video, ${ }^{118}$ leading to accurately demixed neural signal. In contrast, the ${ }^{1190}$ Normcorre registered video displays some residual motion ${ }^{119}$ which leads to relatively poorer extraction of signal. The high ${ }^{192}$ residual signal left by Normcorre+NMF is indicative of poor ${ }^{193}$ signal extraction.

\subsection{Simulation registration video (VIDEO LINK)}

Simulated data registration results. Top row: The video rrior $^{119}$ to registration (left), after dNMF based registration (middle) ${ }^{119}$ and after Normcorre [Pnevmatikakis and Giovannucci, 2017] registration. 2nd Row: Absolute value of the video frames subtracted from the first frame prior to registration (left), after dNMF based registration (middle) and after Normcorre ${ }^{1201}$ (right). If registration is perfect, this frame will look like $\mathrm{a}_{1202}$ weighted sum of Gaussian shapes, one for each cell ( corre $_{\text {i203 }}$ sponding to the cell dimming and brightening, but remaining in place); imperfect registrations are indicated by "spreading" or "doubling" of the cell shapes, as indicated by red arrows. 3rd row: The estimated deformation field at each time point. Bottom row: The locations of the cells across time (colors denote different times) superimposed on the first frame prior to registration (left), after dNMF based registration (middle) and after Normcorre (right). In this video, we see a near perfect stabilization of motion by dNMF, whereas Normcorre displays residual motion as indicated by the absolute residuals (2nd row) as well as the cell spreads in the 4th row.

\subsection{Worm demixing video (VIDEO LINK)}

C. elegans video demixing results. Left block: In the top left we see the $C$. elegans tail video to be demixed. This video involves about 40 neurons of a semi-immobilized worm tail exhibiting calcium activity. In the right we see the reconstruction of this video using the moving spatial components extracted with dNMF. The top row of the bottom panels show a selection of cells zoomed and centered on the motion tracking position inferred by dNMF and in the bottom row is the corresponding spatial component with intensity proportional to the extracted activity level. Note that the neuron centered panels exhibit little motion, hinting at the successful tracking of objects. Right block: This block has the same conventions as the left block but with Normcorre [Pnevmatikakis and Giovannucci, 2017] motion corrected video. Bottom panel: This panel shows the raw video with tracking markers superimposed on top of the neuron centers that are used for ROI averaging. In this video, we see that the large motion exhibited by the worm islargely stabilized by dNMF, but less so by Normcorre (as is particularly visible in the zoomed panels).

\subsection{Worm registration video (VIDEO LINK)}

C. elegans video registration results. Top row: Video prior to registration (left), after dNMF based registration (middle) and after Normcorre [Pnevmatikakis and Giovannucci, 2017] (right) with cell tracking markers superimposed in red. Intensities are oversatured here for better visualization of cell locations. (The flashing blobs in the top left indicate non-neural objects in the FOV such as gut cells.) Middle row: The actual intensity profiles of the videos prior to registration (left), after dNMF based registration (middle) and after Normcorre (right). Bottom row: The positions of cells over time superimposed on the first registered frame for unregistered video (left), after dNMF based registration (middle) and Normcorre (right). Tighter cell groups indicate registration that corrects for motion well. Dispersed cell centers indicate residual local motion. In this video, we see that dNMF has slightly better registration performance than Normcorre as exhibited by the smaller cell spread in several neurons, especially the neurons in the pre-anal ganglion (towards the left border of the frame).

\section{Appendix}

\subsection{Spatial component: non-parametric model (addi- tional details)}

Similar to the standard NMF models, we can parameterize $\boldsymbol{A}$ using an $d$ by $k$ matrix where $d$ is the number of pixels of one time frame of the video and $k$ is the number of objects that are present. $\boldsymbol{f}_{\boldsymbol{\beta}_{t}}(\boldsymbol{A})=\boldsymbol{T}_{t} \boldsymbol{A}$ where $\boldsymbol{T}_{t}: \mathbb{R}^{d \times d}$ and

$$
\boldsymbol{T}_{t}[i, j]=\exp \left(\frac{\left\|\boldsymbol{\beta}_{t} \Psi\left(\boldsymbol{x}_{j}\right)-\boldsymbol{x}_{i}\right\|_{2}^{2}}{2 \sigma^{2}}\right),
$$

where $\beta_{t}$ is a 3 by 10 matrix, and $\Psi: \mathbb{R}^{3} \rightarrow \mathbb{R}^{10}$ is the polynomial basis function that maps a $3 \mathrm{D}$ location into its 10-dimensional quadratic representation in the following way 
$\Psi\left([x, y, z]^{T}\right)=\left[1, x, y, z, x^{2}, y^{2}, z^{2}, x y, y z, z x\right]^{T}$. The choice ${ }_{1213}$ of $\sigma$ controls the amount of the spread of the mass of a pixel ${ }_{1214}$ into nearby pixels. If $\sigma$ is large then each pixel will diffuse ${ }_{215}$ into many pixels around it which will create artificial over ${ }_{\uparrow 216}$ lap between the component footprints. If $\sigma$ is very small $1_{1217}$ then we get vanishing gradients. A careful choice of $\sigma$ is 1218 therefore important for the optimization. We usually set the $\mathbf{1}_{219}$ $\sigma$ to 0.1 in our experiments. If we set the $\boldsymbol{\beta}_{1: T}$ and $\boldsymbol{T}_{1: T^{1220}}$ to the identity we will recover the original formulation of $\mathrm{f}_{121}$ NMF [Lee and Seung, 2001]. However, if we optimize over 1222 $\boldsymbol{\beta}_{1: T}$ we can capture the nonlinear deformation of the objects1223 and estimate the time varying signals more accurately. Furthermore, using this type of kernel matrix as a surrogate for the quadratic transformation has the advantage that it allows us to compute gradients of the cost function with respect to $\beta_{t}$ :

$$
\begin{aligned}
& \frac{\partial \mathcal{L}}{\partial \boldsymbol{\beta}_{t}}=\sum_{i, j=1}^{d} \frac{\partial \boldsymbol{T}_{t}[i, j]}{\partial \boldsymbol{\beta}_{t}} \frac{\partial \mathcal{L}}{\partial \boldsymbol{T}_{t}[i, j]} \\
& =-\boldsymbol{G}_{t}[i, j]\left(\frac{\boldsymbol{\beta}_{t} \Psi\left(\boldsymbol{x}_{\boldsymbol{j}}\right)-\boldsymbol{x}_{i}}{2 \sigma^{2}}\right) \boldsymbol{T}_{t}[i, j] \\
& \text { where } \quad \boldsymbol{G}_{t}=\frac{\partial \mathcal{L}}{\partial \boldsymbol{T}_{t}}=\left(\boldsymbol{T}_{t} \boldsymbol{A} \boldsymbol{C}_{t}-\boldsymbol{Y}_{t}\right)\left(\boldsymbol{C}_{t}^{T} \boldsymbol{A}^{T}\right)
\end{aligned}
$$

We can also compute the gradient with respect to other parameters of the model similar to the vanilla NMF and derive the optimal step size for faster multiplicative updates:

$$
\begin{aligned}
& \frac{\partial \mathcal{L}}{\partial \boldsymbol{C}}=\sum_{t=1}^{T}\left(\boldsymbol{T}_{t} \boldsymbol{A}\right)^{T} \boldsymbol{T}_{t} \boldsymbol{A} \boldsymbol{C}-\left(\boldsymbol{T}_{t} \boldsymbol{A}\right)^{T} \boldsymbol{Y}_{t} \\
& \quad+\lambda_{C}\left(2 \boldsymbol{C}_{t}-\boldsymbol{C}_{t-1}-\boldsymbol{C}_{t+1}\right) \\
& \frac{\partial \mathcal{L}}{\partial \boldsymbol{A}}=\sum_{t=1}^{T} \frac{\partial \boldsymbol{T}_{t} \boldsymbol{A}}{\partial \boldsymbol{A}} \frac{\partial \mathcal{L}}{\partial \boldsymbol{T}_{t} \boldsymbol{A}}=\sum_{t=1}^{T} \boldsymbol{T}_{t}^{T} \boldsymbol{A}\left(\boldsymbol{T}_{t} \boldsymbol{A} \boldsymbol{C} \boldsymbol{C}^{T}-\boldsymbol{Y}_{t} \boldsymbol{C}_{t}^{T}\right)
\end{aligned}
$$

example, the $k$ th column of $\boldsymbol{D}$ has 1's in the entries that correspond to plausible pixel locations that the $k$ th neuron may occupy. Alternatively, $\boldsymbol{D}$ can contain weighted values such that pixels with smaller distance values to a component will tend to have a larger weights.

The utilization of this spatial location regularization using statistical atlases (along with the corresponding strong prior information on the number of cells that should be visible in the field of view) is an important advantage of dNMF (and loca-NMF [Saxena et al., 2019]) over blind deconvolution techniques such as CNMF [Pnevmatikakis et al., 2016].

\subsection{Regularization: spatial location priors}

One of the key advantages of our modular framework is that, if available, we can use statistical atlases of neural positions to better condition our non-convex objective function towards favorable local minima. This is especially useful in the nonparametric modeling of spatial components, where we may not have a good model of the spatial occupancy map of cells.

Similar to the approach taken by [Saxena et al., 2019], we can introduce a regularizer to encourage spatial proximity of non-parametric components to a set of predefined centers that is defined by the statistical atlas of neuron positions. Given a (non-negative) constraint matrix $\boldsymbol{D} \in \mathbb{R}^{d \times k}$ such that each column encodes the allowable occupancy maps of each of the $k$ cells, the regularizer is:

$$
\mathcal{R}_{S}(\boldsymbol{A})=\lambda_{S} \sum_{i j} \boldsymbol{A}_{i j} \boldsymbol{D}_{i j}
$$

The constraint matrix $\boldsymbol{D}$ determines the span by which pixels can be part of a spatial footprint for each component. For 